Apidologie, 1977, 8 (2), 169-204.

\title{
VERGLEICHENDE UNTERSUCHUNGEN DER RÜSSELLÄNGE VON ARBEITERINNEN, KÖNIGINNEN UND DROHNEN DER HONIGBIENE (APIS MELLIFICA L.)
}

\author{
Étude comparative de la longueur du proboscis des ouvrières, \\ des reines et des mâles de l'abeille (Apis mellifica L.)
}

\section{F.-K. BÖTTCHER,}

Bayer. Landesanstalt für Bienenzucht,

Burgbergstr. 70 ,

D.8520 Erlangen

\author{
SUMMARY \\ COMPARATIVE STUDIES ON PROBOSGIS LENGTH OF \\ HONEYBEE WORKERS, QUEENS AND DRONES \\ (Apis mellifera L.)
}

A great many measurements of the proboscis of the three castes (workers, queens, drones) of the geographic races Apis mellifera L., A.m. ligustica Spin., A.m. carnica Pollm. and A.m. caucasica Gorb. showed :

1. In the four examined races the proboscises of queens and drones were about equal in length : Mellifera 4,144/4,042; Ligustica 4,056/4,085; Carnica 4,236/4,179; Caucasica 4,355/ $4,228 \mathrm{~mm}$.

2. Except for one irregularity in the Italian race, the lengths of proboscis approximately corresponded in the ascertained averages as well as in the total dispersion to those of the workers of their race. In the quoted sequence of races, however, they increased to a somewhat less degree than the proboscis of workers. That means proboscis length is influenced not only by sexual and caste determinatin factors but also by race determinating factors.

3. When rearing queens under the same environmental conditions of Carniolan and Caucasian colonies with workerbees of different proboscis lengths, a significant difference in the proboscis length of queens of both races was to be stated in a few individuals already, even if the proboscis length of their sister workers did not differ much.

4. Within the various races, a correlation of proboseis length of workers and sexual individuals mostly could not be established. Rearing of a greater number of queens under the same conditions would be necessary for clarifying this problem. 
5. The previous results indicate that measuring the proboscis length of queens may be a useful aid in the selection out of cross breedings of strains with long proboscises.

\section{ZUSAMMENFASSUNG}

Zahlreiche Messungen der Rüssel der 3 Bienenkasten (Arbeiterinnen Königinnen und Drohnen) bei den geographischen Rassen Apis mellifica mellifica L., A.m. ligustica Spin., A.m. carnica Pollm. und A.m. caucasica Gorb. ergaben :

1. Bei den vier untersuchten Rassen waren die Rüssel der Königinnen und der Drohnen ungefähr gleich lang : Mellifica 4,144/4,042, Ligustica 4,056/4,085, Carnica 4,236/4,179, Caucasica $4,355 / 4,228 \mathrm{~mm}$.

2. Die Rüssellängen entsprachen sowohl in den gefundenen Mittelwerten (mit Ausnahme einer Unregelmäßigkeit bei der Ligustica-Rasse) wie in der Gesamtverteilung ungefähr denen der Arbeitsbienen jeder Rasse. Sie nahmen jedoch in der genannten Rassenreihe in etwas geringerem Maße zu als die Rüssel der Arbeitsbiene. Das besagt, daß die Rüssellänge nicht nur von geschlechts- und kastendeterminierenden, sondern auch von rassebestimmenden Faktoren beeinflußt wird.

3. Bei Aufzucht von Königinnen aus Carnica- und Caucasica-Völkern von verschiedener Rüssellänge der Arbeitsbienen unter gleichen Bedingungen zeigte sich ein klarer Unterschied in der Rüssellänge der Königinnen beider Rassen schon bei wenigen Tieren, und zwar selbst bei nur geringen Längenunterschieden der Rüssel ihrer Arbeiterschwestern.

4. Innerhalb der einzelnen Rassen war eine Korrelation zwischen der Rüssellänge von Arbeitsbienen und Geschlechtstieren zumeist nicht festzustellen. Aufzucht in größerer AnzahI unter gleichen Bedingungen wäre zur weiteren Klärung dieser Frage erforderlich.

5. Die bisherigen Ergebnisse sprechen dafür, daß die Messung der Rüssellänge der Königinnen zur Selektion langrüsseliger Bienenstämme aus Rassekreuzungen eine brauchbare Hilfe sein dürfte.

Die Länge des Rüssels der Arbeiterinnen der verschiedenen geographischen Bienenrassen ist schon vielfach untersucht worden (z. B. von Сноснцоv 1916, Michailov 1926, Alpatov 1929, Skorikov 1929, Orösi-Pál 1934, Farcas 1939, Goetze 1940, Giavarini 1953, Vlatkovic und Kulincevic 1958, Gromisc 1961). Bei der Deutschen oder Dunklen Biene (Apis mellifica mellifica L.) ist sie mit 5,8-6,4 mm am kürzesten, bei der Kaukasischen Biene (Apis mellifica caucasica Gorb.) mit 6,6-7,1 mm bei Einzelwerten bis 7,5 mm am längsten. Dazwischen liegen die Rüssel der Italiener Biene (Apis mellifica ligustica Spin.) mit 6,4-6,7 mm (Alpatov 1929 gibt dafür 6,15-6,45 mm an), und die der Kärntner Biene (Apis mellifica carnica Pollm.) mit 6,5-6,8 mm. Ihre Länge wird sehr von den wechselnden Außenbedingungen beeinflußt (Michailov 1927).

Besondere Beachtung verdient die Rüssellänge wegen ihrer Bedeutung für die Honigleistung der Bienen im Rotklee (Trifolium pratense L.) und für die Bestäubung seiner Blüten. Bienen mit langem Rüssel, nämlich die der Carnica-, der Ligustica - und der Caucasica-Rasse vermögen den Rotklee häufiger und besser zu nutzen als die kurzrüsseligen Mellifica-Bienen, die nur sehr selten Rotkleehonig bringen. 
Infolge der tiefen Kelche des Rotklees ist sein Nektar gewöhnlich nur den Hummeln mit ihrem außerordentlich langen Rüssel zugänglich. Aber langrüsselige Bienen haben, wenn der Nektar in den röhrenförmigen Kelchen infolge warmer und feuchter Witterung höher steigt, ebenfalls die Möglichkeit, ihn zu erreichen. Vermutlich bleiben außerdem bei Trockenheit die Kelche kürzer, was aber bisher nicht bewiesen werden konnte. Auch mag schon ein leichtes Welken der Blüten das Eindringen der Bienen in die Tiefe ihrer Kelche begünstigen (GoETzE 1948).

Diese Erfahrungen machte man insbesondere, als die in Deutschland ursprünglich einheimische dunkle Biene (Apis mellifica mellifica L.) noch nicht so weitgehend wie heute von der Kärntner Biene (A.m. carnica Pollm.) verdrängt war. Es erregte geradezu Aufsehen, daß einzelne Völker gewöhnlich im Monat August sehr viel Honig, die meisten anderen hingegen zur selben Zeit nichts eingebracht hatten. Stets handelte es sich um den dünnflüssigen, hellen, leicht säuerlich schmeckenden Rotkleehonig, der von langrüsseligen Bienen eingetragen worden war (GoETze 1948). Das kam bei uns alle 3-4 Jahre vor, in denen wir derartige Honigproben von einer ganzen Reihe von Imkern erhielten.

Am stärksten fiel es uns bei Herrn Distel in Fürth/Bay. auf. Während 29 seiner Völker mit Rüssellängen von z.B. durchschnittlich $6,08,6,10,6,12$ und $6,19 \mathrm{~mm}$ im August keinen Honig und im ganzen Jahr im Mittel überhaupt nur 2,5 kg brachten, gab ein einziges, ein Carnica-Volk mit der Rüssellänge von durchschnittlich $6,59 \mathrm{~mm}$, nicht weniger als $30 \mathrm{~kg}$ ! Die pollenanalytische Untersuchung bestätigte den äußeren Befund, daß es sich bei dem im August geernteten Hauptanteil um reinen Rotkleehonig handelte.

Im gleichen Jahr hatte Herr Hörner, der die Carnica-Biene in der Nähe von Erlangen züchtet, eine sehr gute Honigernte, vor allem vom Rotklee, von 15,6-29,7 kg je Volk. Lediglich 3 Völker waren ganz ohne Ertrag aus dieser Tracht geblieben. Die Rüssellänge der Arbeitshienen betrug bei den ertragreichen $6,48-6,70 \mathrm{~mm}$, bei den ertraglosen hingegen $6,19,6,25$ und $6,35 \mathrm{~mm}$.

Ebenfalls Rotkleehonig lieferten Völker von Herrn Dr. Thiем, Roth, mit einer Rüssellänge der Arbeiterinnen von 6,60 und $6,50 \mathrm{~mm}$. Versager im Rotklee waren die mit einer kurzen Rüssellänge von $6,19,6,25$ und $6,35 \mathrm{~mm}$.

Sicher spielen beim Sammeln von Rotkleenektar neben der Rüssellänge auch die Findigkeit und Aktivität der Bienen eine Rolle (Goetze 1956). Aber die besten dieser Eigenschaften nützen nichts, wenn der Rüssel zu kurz ist, um den Nektarspiegel zu erreichen. Der lange Rüssel ist somit die erste Voraussetzung für den größeren Honigertrag. Deshalb muß man bei der Züchtung einer “ rotkleefähigen » Biene vor allem auf ihre Rüssellänge Wert legen. Für die Nutzung anderer Trachten hat die Rüssellänge indessen keine Bedeutung (HeJtmaneK 1961). 
Aber wichtig ist sie für die Bestäubung der Rotkleeblüten und somit für den Samenertrag. Denn Bienen, die den Nektar erlangen können, werden die Blüten eher besuchen und deren Pollen häufiger übertragen als kurzrüsselige, die nicht vom Nektar zu den Blüten gelockt werden (vaN LAERE und Martens 1962).

So hat die Rüssellänge der Arbeitsbienen sowohl für die Imkerei wie für den Samenbau große Bedeutung, und manches ist schon versucht worden, sie züchterisch zu fördern (Goetze 1930, 1940, 1956). Dabei ging man bisher stets nur von der Rüssellänge der Arbeitshienen aus, denn der Rüssel der Geschlechtstiere ist um etwa ein Drittel kürzer. Es wäre aber sehr wichtig, die Selektion auch nach dem Rüssel der Königinnen und Drohnen treffen zu können, und nicht nach dem der nur bedingt fortpflanzungsfähigen Arbeitsbienen. Das würde sie wesentlich erleichtern.

\section{FRAGESTELLUNG}

Über die Länge des Rüssels von Königinnen und Drohnen ist bisher nur wenig bekannt. Einige Daten liegen von Kaukasischen Königinnen und Drohnen vor (Lekishvili und Khideshelr 1967), insbesondere auch üher die Abhängigkeit der Länge der Drohnenrüssel von der Rasse des Pflegevolkes (Melnichenko 1962).

Vor allem wissen wir noch nicht, wie weit die Länge des Königinnenund Drohnenrüssels der Länge des Rüssels der Arbeitsbienen entspricht. Da die Königinnen ebenso wie ihre schwesterlichen Arbeitsbienen aus befruchteten Eiern hervorgehen, also im ganzen das gleiche Erbgut haben, müßte die Längenausbildung ihres Rüssels mit der des Rüssels ihrer Arbeiterschwestern parallel laufen. Und da die Drohnen aus unbefruchteten Eiern entstehen, wäre zu erwarten, daß sich bei ihnen das Erbgut « Rüssellänge » ihrer Mutter unverdeckt widerspiegelt.

In dieser Arbeit werden die Längenwerte von Rüsseln gebracht, die wir während einiger Jahre an unseren Carnica-Zuchtköniginnen, - Drohnen und - Arbeitsbienen, dazu auch an dem uns zugänglichen Caucasica-, Ligustica - und Mellifica-Material gewinnen konnten. Außerdem wird das Ergebnis der Rüsselmessungen bei lang-und kürzerrüsselig veranlagten unter gleichen Bedingungen aufgezogenen Königinnen dargestellt.

\section{METHODE}

Die Rüssel wurden zumeist an den lebenden mit Kohlensäure betäubten Bienen gemessen (Bötтcher 1961, 1975). Man arbeitet bei diesem Verfahren 
sehr schnell, weil das Herauspräparieren des Rüssels entfällt. Außerdem bietet diese Methode den für die züchterische Praxis wichtigen Vorteil, daß sich die auf Rüssellänge ausgelesenen Königinnen, Drohnen und auch Arbeitsbienen selbst zur Zucht verwenden lassen. Tiere, die wir hierzu nicht brauchten, haben wir auch mittels Äther abgetötet.

Sie kommen in ein kurzes, sich nach vorn verengendes Röhrchen, welehes man hinten mit einem durchbohrten Stopfen oder einem Stück Schaumstoff (Moltopren) abschließt. Von hier aus führt ein dünner Schlauch (z.B. ein Fahrradventilschlauch) über eine Waschflasche zu einer Kohlensäureflasche, aus der man zur Betäubung der Biene einen leichten Gasstrom einleitet. Danach streckt sie ihren Rüssel, der mit dem Kopf aus dem Röhrchen herausragen soll.

Unter dem Binokular erfaßt man den Rüssel mit einer feinen Pinzette und zieht ihn vorsichtig nach vorn, so daß sich die Zügel (Cardo) und Angeln (Lorum) spannen und das Submentum sich mit dem Mentum in eine Ebene legt. Um ihn in dieser Lage zu halten, legt man seine Spitze mittels einer kleinen beschwerten Glasplatte auf einem mit Fließpapier beklebten Gummiblock fest. Man kann ihn nun mit der gleichen Genauigkeit messen wie nach dem Herauspräparieren und Einbetten in Wasser zwischen Objektträger und Deckglas (vgl. Bötrcher 1962/1965, 1975). Es scheint jedoch, daß bald nach dem Schlüpfen mit Kohlensäure betäubte Königinnen zu einem etwas größeren Teil als gewöhnlich auf der Belegstelle verloren gehen. Deshalb nahmen wir die Rüsselmessungen in einigen Fällen erst nach der Begattung der Königinnen vor.

Manche Drohnen strecken bei der Betäubung mit Kohlensäure ihren Rüssel nicht. Er erscheint verkrampft. Auf sie muß man zur Messung verzichten. Wo es angängig ist, kann man die Drohnen (ebenso wie die Königinnen und Arbeitsbienen) auch mit Äther betäuben, was die Rüsselstreckung stets bewirkt, bei reifen Drohnen jedoch auch das Ausstülpen ihres Geschlechtsapparates und damit ihren Tod zur Folge hat.

\section{MATERIAL}

\section{1. - Apis mellifica mellifica L.}

Reine Mellifica-Bienen sind heute bei uns nur noch schwer zu finden. Die meisten Bienen unseres Landes sind verkreuzt. Dennoch konnten einige Völker mit den Merkmalen dieser Rasse auf Rüssellänge ihrer Arbeitsbienen und zum Teil auch ihrer Geschlechtstiere untersucht werden. Die von ihnen stammenden Königinnen mußten jedoch, bis auf einzelne Schwarmköniginnen, in Carnica-Völkern aufgezogen werden. 


\section{2. - Apis mellifica carnica Pollm.}

Die Kärntner Biene wird in Deutschland heute seit dem 2. Weltkrieg allgemein gehalten. Infolge Vermischung mit der heimischen A.m. mellifica L. ist sie aber oft nicht ganz rein.

Da wir ständig Carnica-Königinnen ziehen, standen diese uns von unseren jeweiligen Zuchtvölkern ebenso wie die Drohnen und Arbeitsbienen in genügend großer Zahl zur Verfügung. Sie stammen von 14 verschiedenen Völkern, die in ihren Merkmalen und Eigenschaften der Carnica-Rasse voll entsprachen, also als reinrassig gelten können.

\section{3. - Apis mellifica ligustica Spin.}

Die Italiener Biene wurde in den dreißiger Jahren in Rotkleesamenbaugebiet am Niederrhein von dem Züchter Kleinewegen in Sevelen mit guten Erträgen vom Rotklee gehalten (Gostze 1948). Heute findet man sie in Deutschland nur noch gelegentlich.

Unser Ligustica-Material (8 Völker) stammte z.T. aus Italien, und zwar von dem Züchter Piana, Castel San Pietro, Bologna, z.'T. aus den USA. Im letzten Fall handelte es sich um die c Star-Line » des Züchters Jesupp in Georgia und um Material eines unbekannten amerikanischen Züchters.

\section{4. - Apis mellifica caucasica Gorb.}

Die echten wegen ihrer extremen Langrüsseligkeit bekannten Kaukasier Bienen aus der UdSSR werden bei uns nicht gehalten. Sie entwickeln sich hier in der Regel nur zu mäßig starken Völkern. Wir erhielten OriginalKaukasier Königinnen zumeist unmittelbar aus der Sowjetunion, z.T. auch über Frau Prof. Meyerhoff, Hohenneuendorf, Herrn Dr. Oschmann, Thälermühle, Herrn Dr. Drener, Mayen und Herrn Prof. Bornus, Polen. Allen danke ich bestens. So konnte ich Rüsselmaterial von 12 verschiedenen echten Kaukasier-Völkern messen. Außerdem untersuchte ich amerikanische Herkünfte, die jedoch allgemein etwas kurzrüsseliger sind. Sie werden in Deutschland hier und da vermehrt. Von den Original Kaukasier-Völkern Drohnen und Schwarmköniginnen zu bekommen, war infolge ihrer mäßigen Entwicklung schwierig. Deshalb sind alle von uns untersuchten Kaukasier Königinnen Nachschaffungsköniginnen, die wir in Carnica-Völkern gezogen haben.

\section{A. - RÜSSELMESSUNGEN BEI BELIEBIGEN ARBEITSBIENEN, KÖNIGINNEN UND DROHNEN DER VERSGHIEDENEN BIENENRASSEN}

Um einen Überblick über die Rüssellänge der 3 Bienenkasten bei den 4 untersuchten geographischen Rassen zu bekommen, habe ich zunächst die 
Ergebnisse aller gemessenen Rüssellängen nach Proben und Rassen summarisch zusammengestellt. Dabei blieb die umweltbedingte Variabilität so gut wie unberücksichtigt.

\section{a) Mittelwerte}

Vorweg sei in Tabelle 1 das Endergebnis, der Durchschnitt der Mittelwerte aller Proben nach Rassen angegeben. Es ist aus den Mittelwerten der einzelnen Völker (Tab. 2-5) berechnet und nach der Länge der Arbeiterrüssel geordnet. In den Tabellen 2-5 findet man außerdem das gewogene Mittel, welches für die statistische Bearbeitung ${ }^{*}$ ) verwendet wurde.

Tав. 1. - Mittlere Rüssellänge von Arbeitsbienen, Königinnen und Drohnen der verschiedenen Rassen

(berechnet aus den mittleren Rüsselwerten der Völker, in $\mathrm{mm}$ ).

TABL. 1. - Longueur moyenne du proboscis d'ouvrières, de reines et de mâles des différentes races

(calculée à partir des valeurs moyennes du proboscis des colonies, en mm).

\begin{tabular}{l|c|c|c} 
& $\begin{array}{l}\text { Arbeiter } \\
\text { Ouvrières }\end{array}$ & $\begin{array}{c}\text { Königinnen } \\
\text { Reines }\end{array}$ & $\begin{array}{c}\text { Orohnen } \\
\text { Mâles }\end{array}$ \\
\hline A.m. mellifica & 6,256 & 4,144 & 4,042 \\
A.m. ligustica & 6,588 & 4,065 & 4,085 \\
A.m. carnica & 6,593 & 4,236 & 4,179 \\
A.m. caucasica USA & 6,695 & 4,183 & 4,133 \\
A.m. caucasica Orig. & 7,119 & 4,355 & 4,228
\end{tabular}

Die Arbeiterrüssel entsprechen den für die 4 geographischen Rassen bekannten eingangs angegebenen Werten : den kürzesten Rüssel haben die Mellifica-, den längsten die Original-Kaukasier Arbeiterinnen. Dazwischen liegen die hier so gut wie gleich langen Rüssel der Carnica- und LigusticaBienen. Bei Untersuchung von noch breiter gestreutem Ligustica-Material könnte sich vielleicht eine Differenzierung ergeben. Die Kaukasier Bienen amerikanischer Herkunft haben kürzere Rüssel als die Original-Kaukasier.

Mit Hilfe des t-Testes wurden die Unterschiede zwischen den verschiedenen Arbeiterrüssellängen geprüft, und zwar die zwischen der Mellificaund Caucasica-, der Mellifica- und Ligustica- sowie der Carnica- und Caucasica-Rasse. Alle Unterschiede waren hochsignifikant $(\alpha<<0,1 \%)$.

* Für Beratung in statistischen Fragen sowie für die Durchführung der erforderlichen statistischen Berechnungen danke ich Herrn Prof. Göır, Herrn Dr. Mautz und Frau Bosch. 


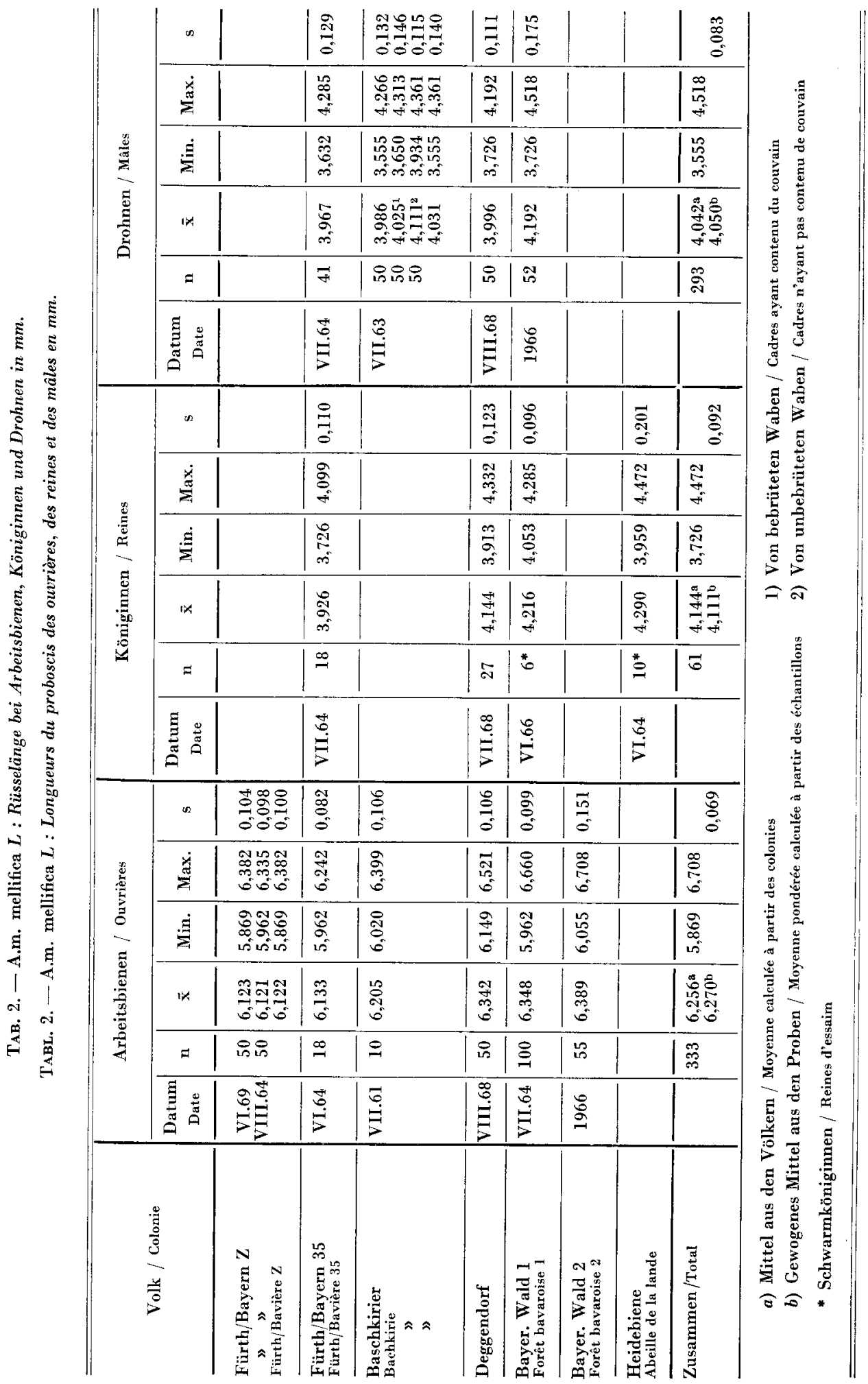


Die Rüssel der Königinnen und Drohnen sind bei den einzelnen Rassen im Mittel ungefähr gleich lang, bei etwas stärkerer Verschiedenheit derer der Kaukasier Rasse. Wie bei den Arbeitsbienen sind sie bei der Mellifica verhältnismäßig kurz, bei der Caucasica lang, und bei der Carnica und Ligustica liegen sie in der Mitte. In diesem Sinne, d.h. der Reihenfolge nach, entspricht die Längenausbildung der Königinnen- und Drohnenrüssel der der Arbeiterrüssel.

Was die Unterschiede der Königinnenrüssel anbetrifft, so erwiesen sich diese zwischen den Rassen Mellifica und Caucasica, Mellifica und Ligustica, Ligustica und Carnica sowie Carnica und Caucasica nach dem t-Test als hochgesichert $(\alpha<<0,1 \%)$, was auch für die übrigen Kombinationen zutreffen dürfte. Dasselbe gilt für die Drohnenrüssel der Rassen Mellifica und Caucasica, Ligustica und Carnica $(\alpha<<0,1 \%)$, sowie Carnica und Caucasica $(\alpha<0,1 \%)$. Gut gesichert sind die Unterschiede der Rüsselmittelwerte der Mellifica- und Ligustica-Drohnen $(1 \%<a<2 \%)$.

Und schließlich sind auch die meist nur geringen Unterschiede zwischen den Mittelwerten der Rüssellängen der Königinnen und Drohnen bei drei von vier untersuchten Rassen statistisch gesichert : Hochgesichert bei den Caucasica- und Mellifica-Geschlechtstieren $(\alpha<<0,1 \%)$, und gut gesichert bei den Carnica-Geschlechtstieren $(1 \%<\alpha<2 \%)$. Lediglich die LigusticaKöniginnen und Drohnen zeigten keinen statistisch abgesicherten Unterschied $(\mathbf{t}=0,82,25 \%<\alpha<50 \%)$. Die hohe Übereinstimmung ihrer Länge kann also Zufall sein.

Aus der Reihe der Rüssel fallen die der Ligustica-Königinnen heraus. Sie sind in ihren Mittelwerten sehr kurz, sogar noch kürzer als die Rüssel der Mellifica-Königinnen. Auch die Rüssel der Caucasica-Geschlechtstiere amerikanischer Herkunft sind zu kurz, um in die Reihe zu passen.

Bei den anderen Rassen findet sich bei näherer Betrachtung eine Eigenheit : Die Rüssellänge der Geschlechtstiere nimmt nicht im gleichen Maße zu wie die der Arbeiterrüssel. Je länger der Rüssel der Arbeitsbienen wird, desto geringer ist die Zunahme des Rüssels der Geschlechtstiere. Oder umgekehrt ausgedrückt : Die bei den Geschlechtstieren gegenüber den Arbeitsbienen bestehende Verkürzung des Rüssels nimmt mit steigender Rüssellänge der Arbeitsbienen der untersuchten Rassen zu.

Die alte Regel : Königinnenrüssellänge $=2 / 3$ der Arbeiterrüssellänge bestätigt sich im vollen Umfange nur bei der Mellifica. Bei allen anderen ist der Rüssel beider Geschlechtstiere kürzer. Dabei zeigen sich zum Teil stärkere Unterschiede. Sie liegen bei den Königinnenrüsseln zwischen 66,24 und $61,17 \%$ der Rüssellänge der Arbeitsbienen und bei den Drohnenrüsseln zwischen 64,61 und 59,39\%, betragen also bis zu 5,07 und 5,22\%. Das sind 7,7\% der Rüssellänge der Mellifica-Königinnen und 8,1\% der Rüssellänge der MellificaDrohnen. 


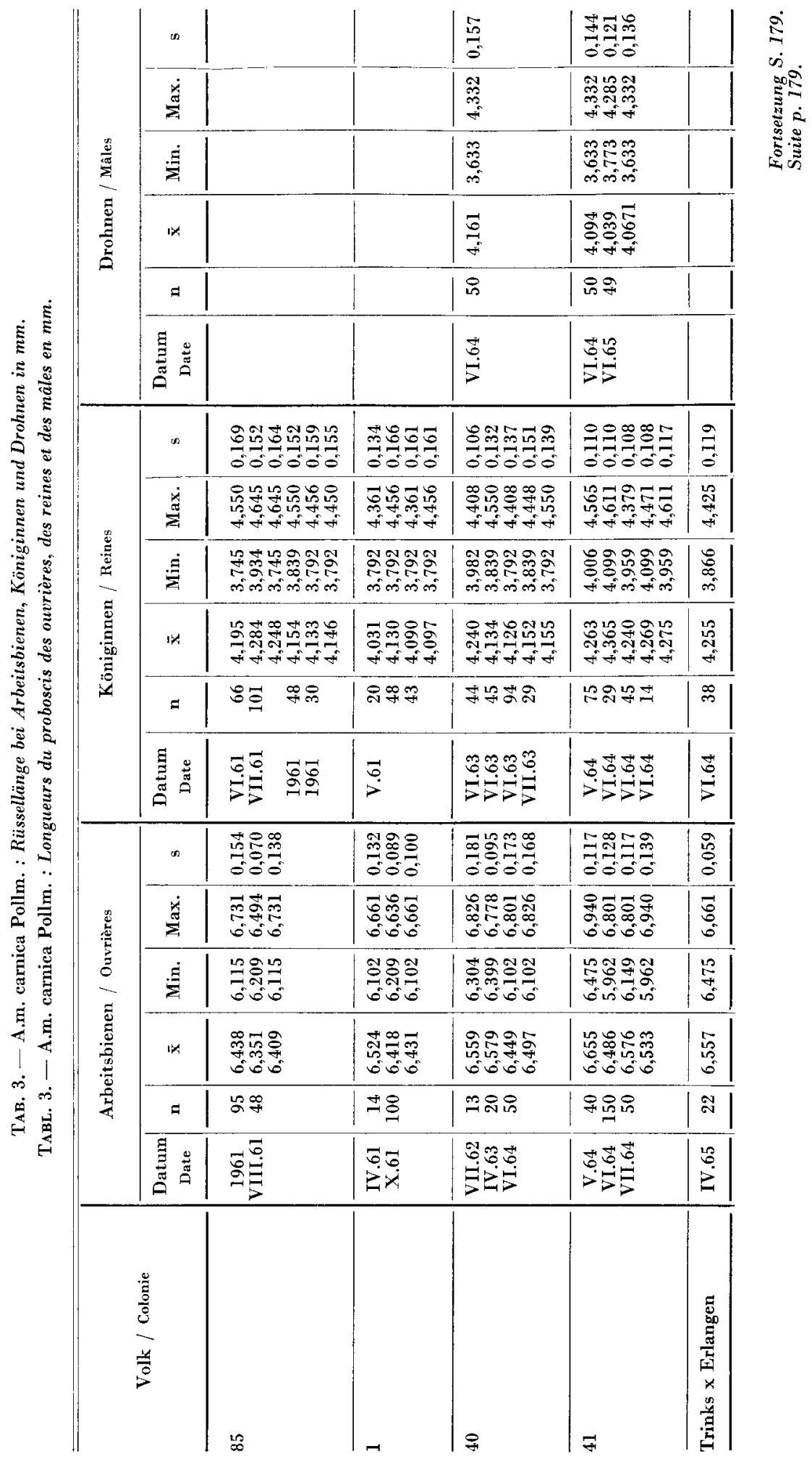


RÜSSELLÄNGE DER 3 KASTEN DER HONIGBIENE

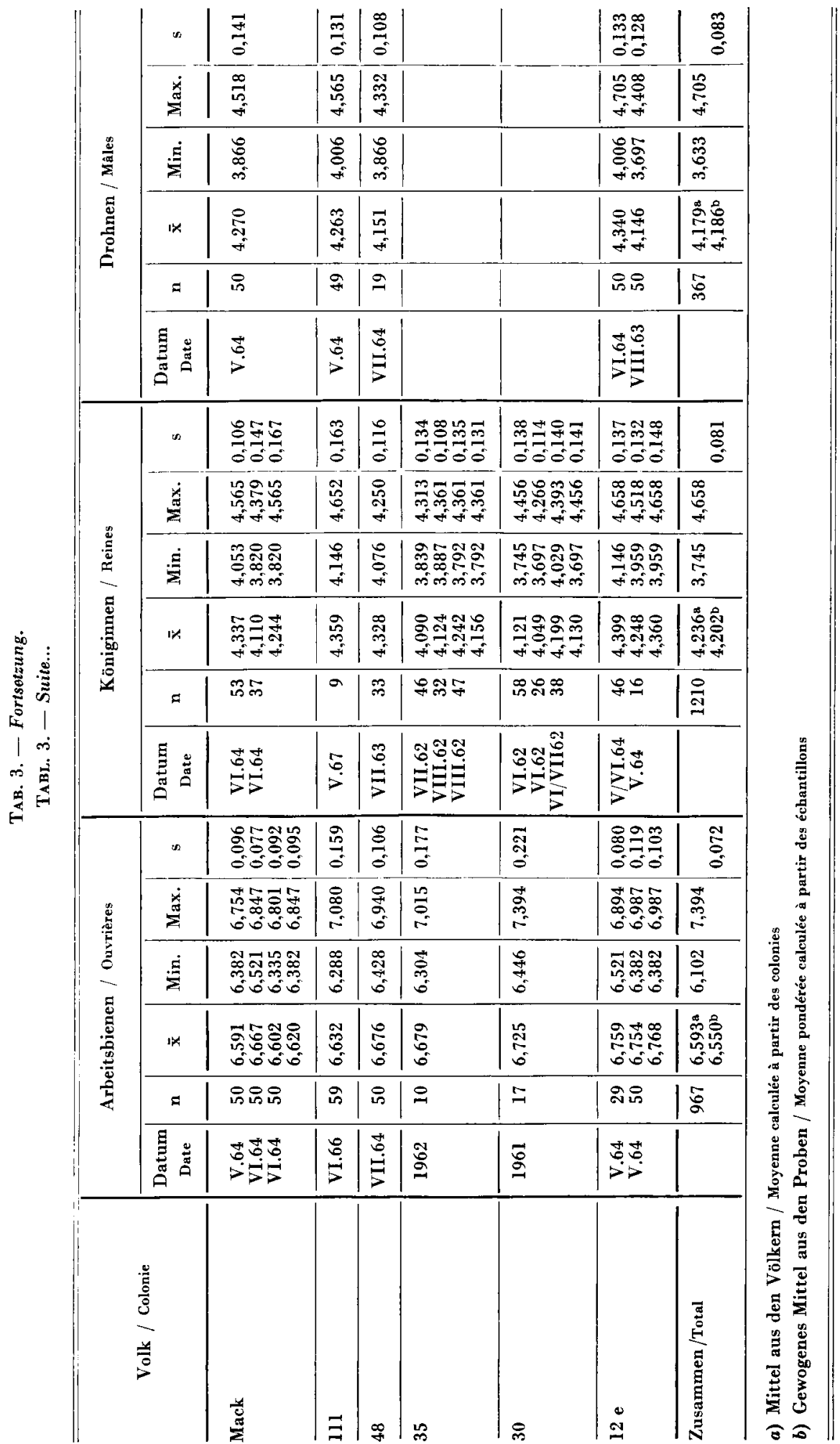




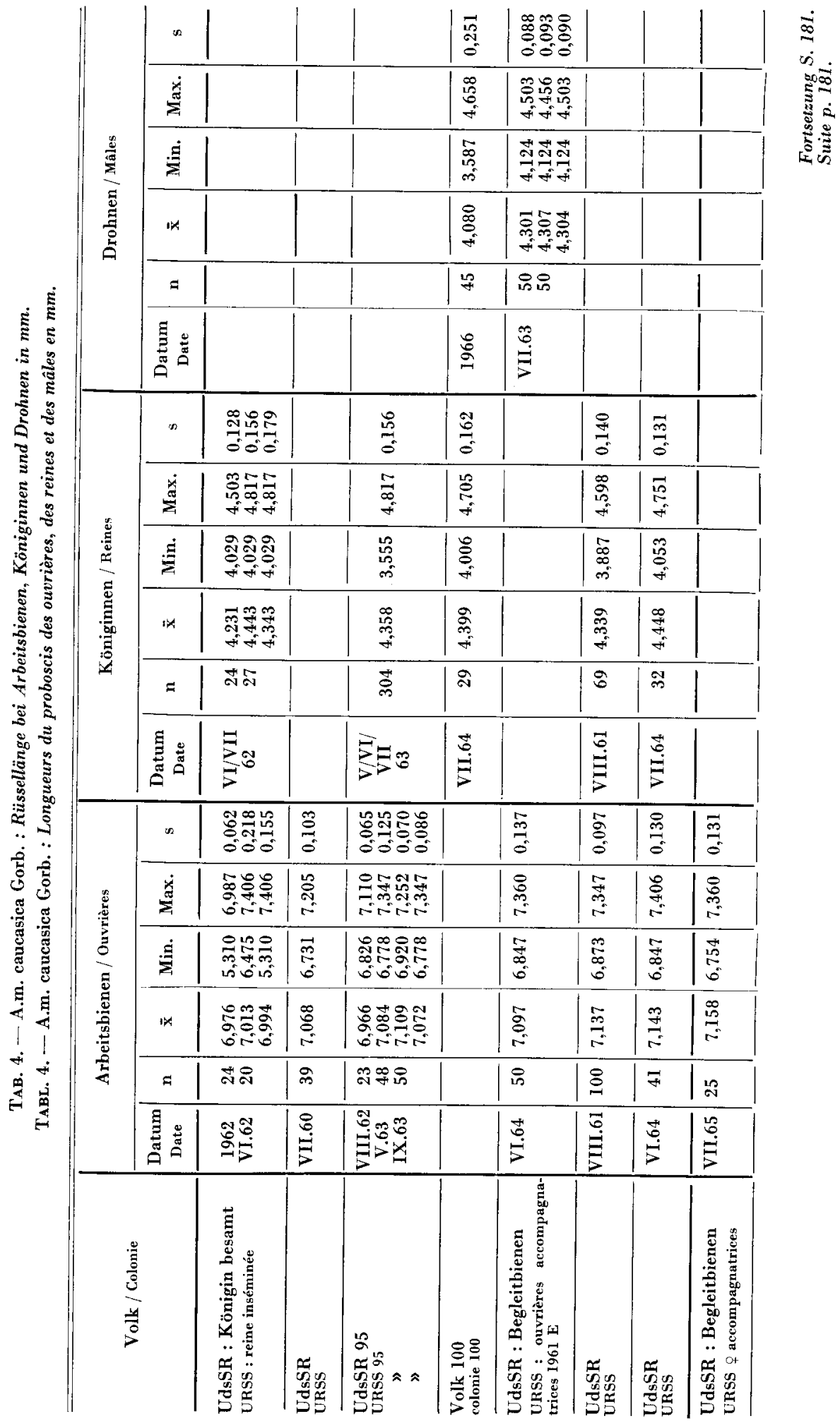




\begin{tabular}{|c|c|c|c|c|c|c|c|c|c|c|c|}
\hline \multirow{6}{*}{ 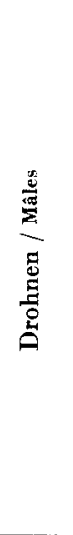 } & n & : & $\mid \frac{\omega}{g}$ & 告 & $\frac{\overrightarrow{0}}{a}$ & & $\stackrel{\text { ga }}{\mathrm{g}}$ & & 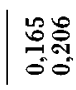 & $\stackrel{\infty}{\sharp}$ & \\
\hline & 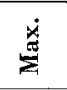 & 䋨 & 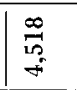 & 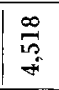 & Ë & & 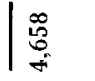 & & 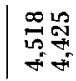 & 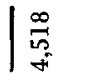 & \\
\hline & $\dot{\Xi}$ & 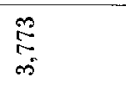 & 鄫 & 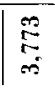 & 总 & & 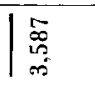 & & 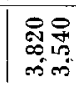 & 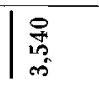 & \\
\hline & $1 *$ & $\stackrel{10}{=}$ & 동 & $\frac{2}{2}$ & శ్ ఫ్ & & 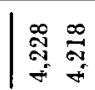 & & 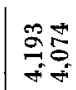 & 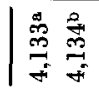 & \\
\hline & $\Rightarrow$ & $\therefore$ & in & 8 & in & & |总 & & 品 & 18 & \\
\hline & 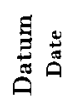 & $\stackrel{8}{5}$ & $\stackrel{8}{\stackrel{0}{5}}$ & & & & & & 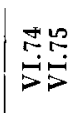 & & \\
\hline \multirow{6}{*}{ 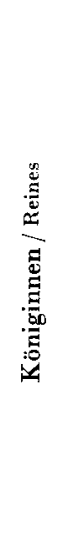 } & $\infty$ & 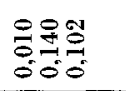 & $\frac{\mathscr{0}}{0}$ & & & : & | & $\left|\begin{array}{l}0 \\
\frac{9}{0} \\
0\end{array}\right|$ & $\begin{array}{l}5 \\
6 \\
0\end{array}$ & 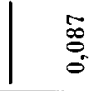 & \\
\hline & 商 & 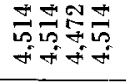 & \begin{tabular}{|l}
$\vec{\alpha}$ \\
$\dot{\alpha}+$ \\
\end{tabular} & & & 总 & 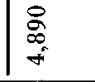 & $\mid$\begin{tabular}{|c}
0 \\
0 \\
5 \\
7
\end{tabular} & \begin{tabular}{|c}
5 \\
5 \\
10 \\
10
\end{tabular} & $\mid \begin{array}{c}\tilde{5} \\
\text { 点 } \\
\text { in }\end{array}$ & \\
\hline & 完 & 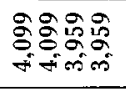 & 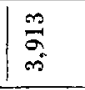 & & & 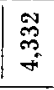 & 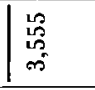 & 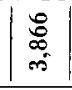 & 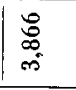 & $\mid$ & \\
\hline & $1 *$ & 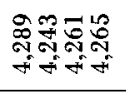 & \begin{tabular}{|l}
$\vec{g}$ \\
g \\
d
\end{tabular} & & & $\stackrel{\infty}{\underset{q}{+}}$ & 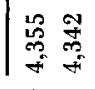 & $\mid$\begin{tabular}{l}
$m$ \\
\hdashline \\
\hdashline
\end{tabular} & 番 & 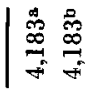 & \\
\hline & $=$ & ริสีลั & in & & & $r$ & \%్త్రి & 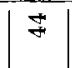 & $\%$ & 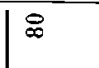 & \\
\hline & 密 & 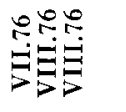 & 蛋 & & & $\because$ & & $\overrightarrow{5}$ & 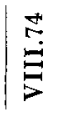 & & \\
\hline \multirow{6}{*}{ 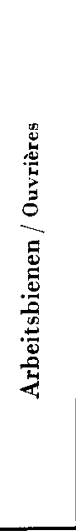 } & $\infty$ & $\begin{array}{l}3 \\
0 \\
0\end{array}$ & $\mid \begin{array}{l}8 \\
0 \\
0\end{array}$ & & & & 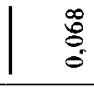 & $\left|\begin{array}{|c|}1 \\
0 \\
0 \\
0\end{array}\right|$ & $\mid \begin{array}{c}0 \\
\vdots \\
0 \\
0\end{array}$ & $\begin{array}{l}\infty \\
\stackrel{\infty}{8} \\
\stackrel{8}{0}\end{array}$ & \\
\hline & 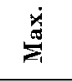 & 总 & 篣 & & & & 番 & $\left|\begin{array}{|c}\vec{\Delta} \\
0 \\
0\end{array}\right|$ & 苐 & $\mid \begin{array}{c}\vec{a} \\
0 \\
0\end{array}$ & 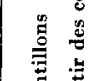 \\
\hline & 这 & 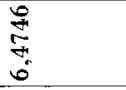 & 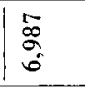 & & & & 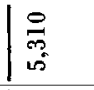 & $\left|\begin{array}{l}7 \\
\vdots \\
0 \\
0\end{array}\right|$ & $\mid \begin{array}{c}7 \\
0 \\
0 \\
0 \\
0\end{array}$ & 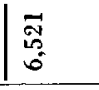 & \\
\hline & $\%$ & 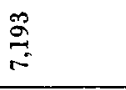 & 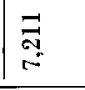 & & & & 噫查 & $\mid$ & 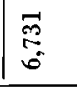 & 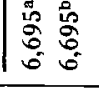 & \\
\hline & $\Rightarrow$ & in & in & & & & | & in & in & $1 \Xi$ & 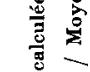 \\
\hline & 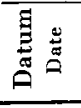 & 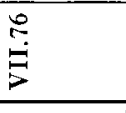 & | & & & & & $\mid$ & \begin{tabular}{|l}
$\vec{E}$ \\
\end{tabular} & & \\
\hline 这 & & 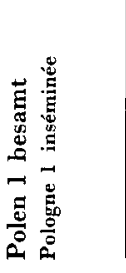 & 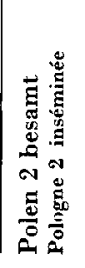 & 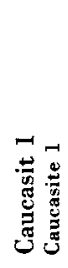 & 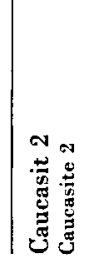 & 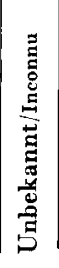 & 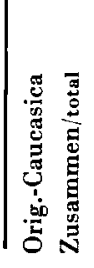 & 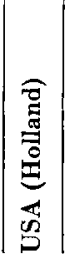 & 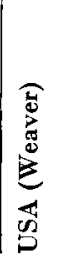 & 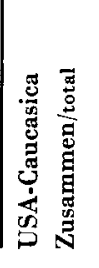 & 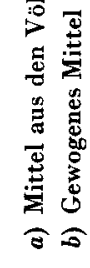 \\
\hline
\end{tabular}




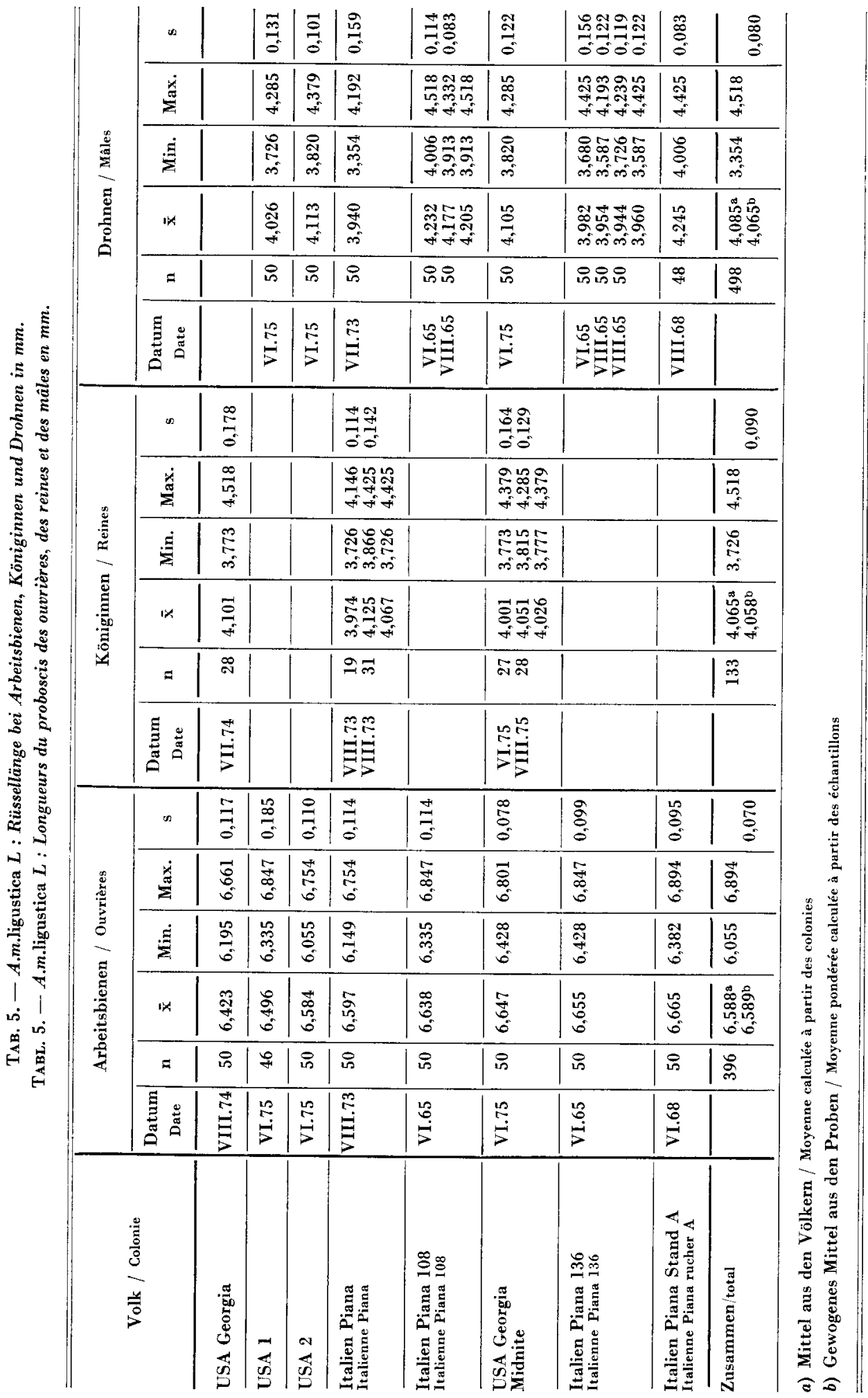




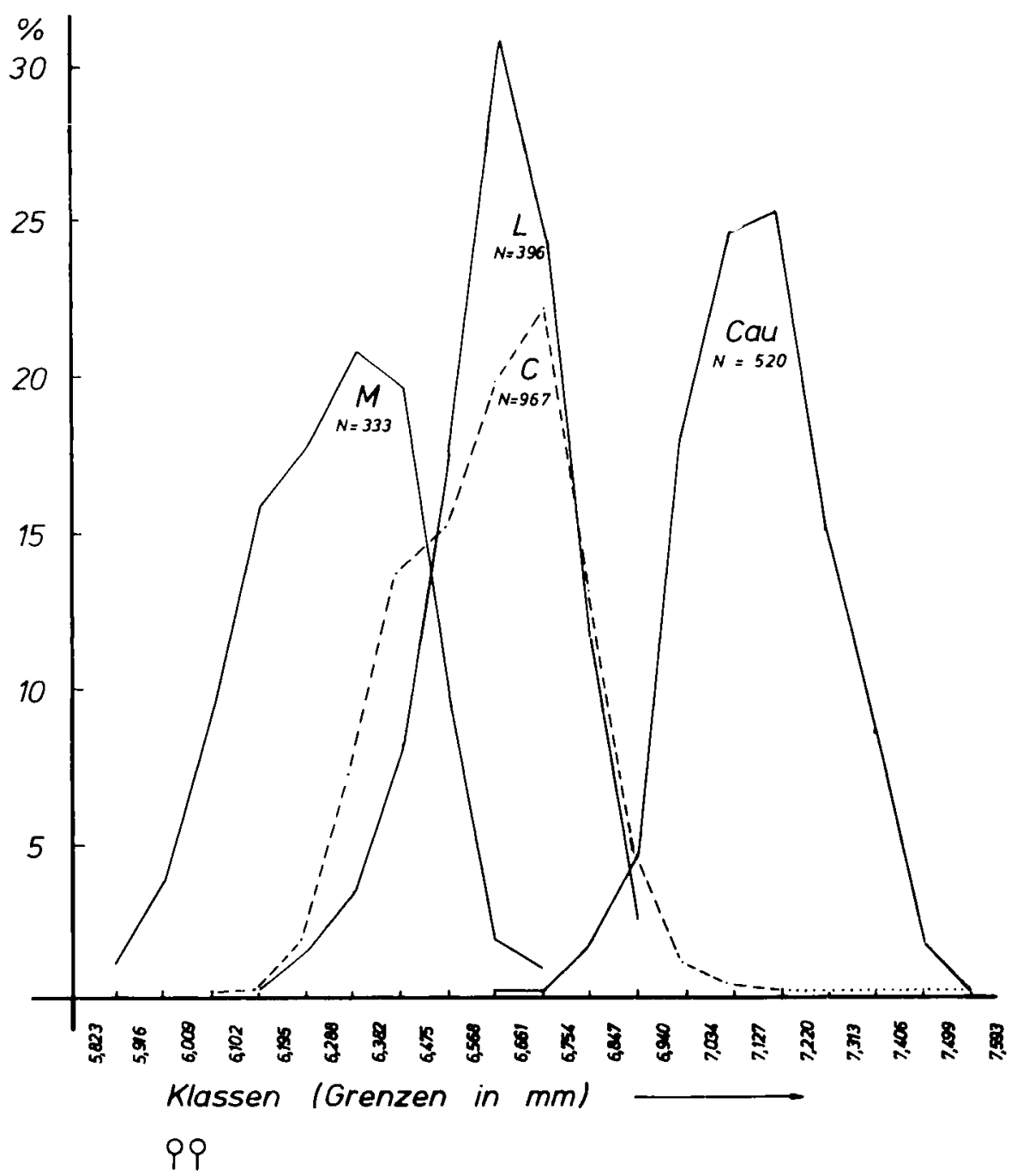

Aвв. 1. - - Rüssellängen der Arbeitsbienen

von A.m. mellifica (M), A.m. carnica (C---), A.m. ligustica (L) und A.m. caucasica (Cau).

Fig. I. - Longueurs du proboscis des ouvrières

d'A.m. mellifica (M), A.m. carnica (C-.-), A.m. ligustica (L) et A.m. caucasica (Cau) 
Das alles besagt, daß die Rüssellänge, offenbar genetisch gesteuert, sich viel stärker bei den Arbeiterinnen manifestiert, als bei den Geschlechtstieren, wo sie keine adaptive Bedeutung hat, und daß sie nicht nur von geschlechts- und kastendeterminierenden, sondern auch von rassebestimmenden Faktoren beeinflußt wird.

\section{b) Gesamtverteilung}

Genaueren Aufschluß über den Zusammenhang zwischen den Rüssellängen der 3 Bienenkasten bei den 4 zu vergleichenden Rassen geben die Verteilungskurven aller gemessenen Werte (Abb. 1-7).

Bei den Arbeiterrüsselkurven (Abb. 1) ergibt sich eine so gut wie völlige Trennung der kurzen Mellifica- und der langen Caucasica-Rüssel. Zwischen beiden, jedoch mehr zu den Rüsseln der Mellifica-Rasse hin, mit denen sie sich etwas überlagern, liegen die sich ungefähr deckenden Kurven der Ligusticaund Carnica-Rüssel.

Die Mellifica- und Caucasica-Kurven lassen sich bei entsprechender Verschiebung mit ihnen zur Deckung bringen, d.h. die Variationsbreite ist bei allen 4 Rassen gleich.

Die Rüsselkurven der Königinnen (Abb. 2) und die der Drohnen (Abb. 3) der 4 Rassen zeigen eine ähnliche Anordnung wie die der Arbeitsbienen : Die Rüssel der Caucasica-Geschlechtstiere befinden sich weit überwiegend im langen Bereich, die der Mellifica-Geschlechtstiere vorwiegend im kurzen. Die Rüssel der Carnica- und Ligustica-Geschlechtstiere liegen in der Mitte. Alle Kurven sind dichter zusammengedrängt. Die Rüssel messen ja auch nur 2/3 der Länge der Arbeiterrüssel. Außerdem überlagern sie sich stärker. Hierin zeigt sich eine unverhältnismäßig größere Streuung. Im übrigen decken sich die Kurven der Königinnenrüssel mit denen der Drohnenrüssel bei allen 4 Rassen nahezu (Abb. 4-7), am besten bei der Carnica (Abb. 4) und bei der Caucasica (Abb. 5). Somit ist auch die Variationsbreite die gleiche. Dabei ist die Kurve der Drohnenrüssellänge entsprechend dem vielfach etwas niedrigerem Mittelwert (Tab. 1) zumeist ein wenig nach links, d.h. nach dem kürzeren Bereich verschoben.

Die Kurve der Ligustica-Königinnen ist zweigipfelig (Abb. 6). Das dürfte wahrscheinlich die Folge der verhältnismässug geringen Anzahl von Meßwerten sein, und die Unregelmäßigkeit in der Reihe der Rüsselmittelwerte bei den Ligustica-Königinnen könnte hierin ihre Erklärung finden. Mit dem Carnica-Gipfel deckt sich nur der zweite Gipfel (Abb. 2). Der erste liegt entsprechend dem niedrigen aus der Reihe herausfallenden Mittelwert bereits über dem ersten der beiden Mellifica-Gipfel. 


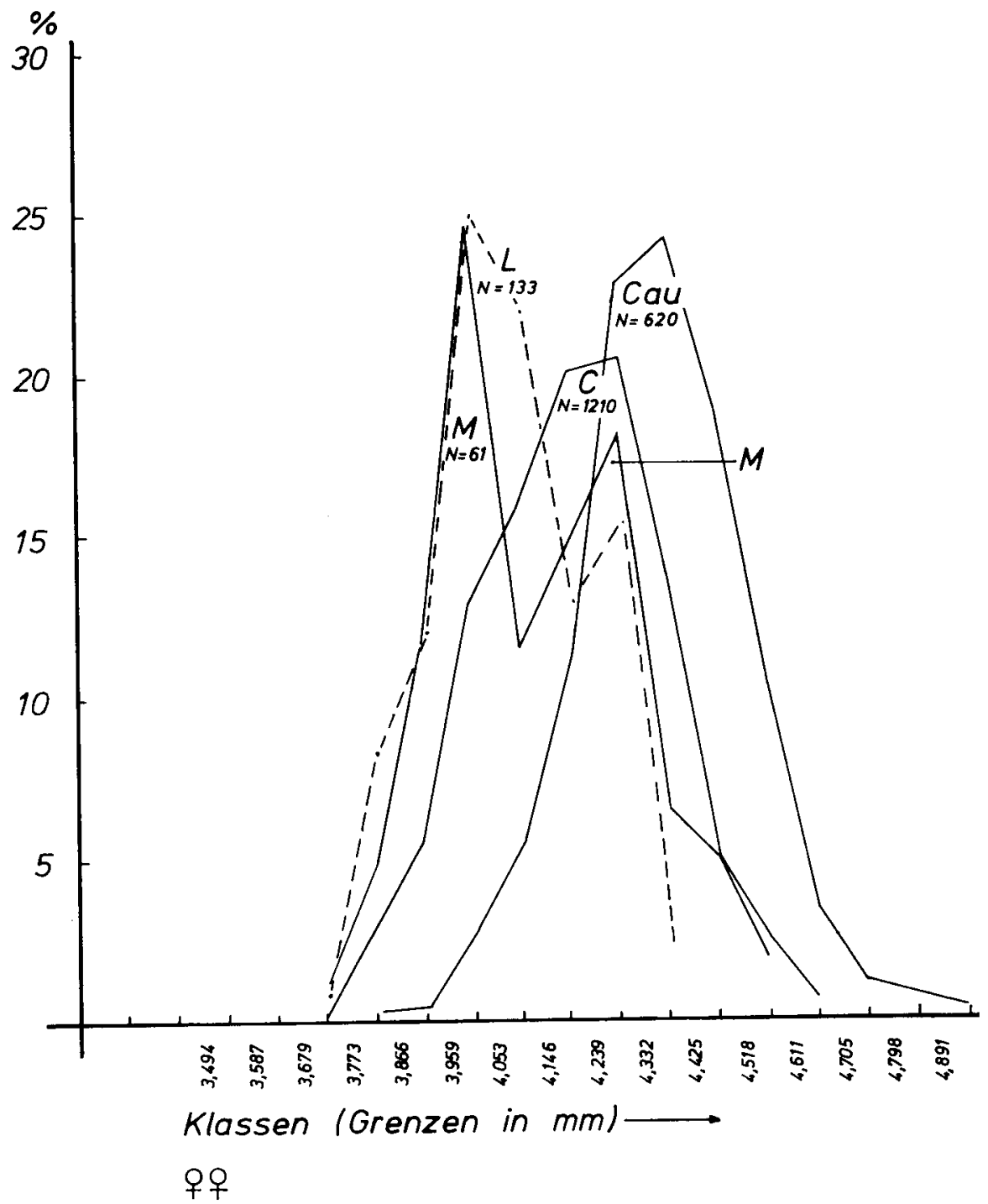

Aвв. 2. - Rüssellängen der Königinnen

von A.m. mellifica (M), A.m. carnica (C), A.m. ligustica (L---) und A.m. caucasica (Cau).

FIg. 2. - Longueurs du proboscis des reines

d'A.m. mellifica (M), A. m, carnica (C), A.m. ligustica (L---) et A.m. caucasica (Cau). 


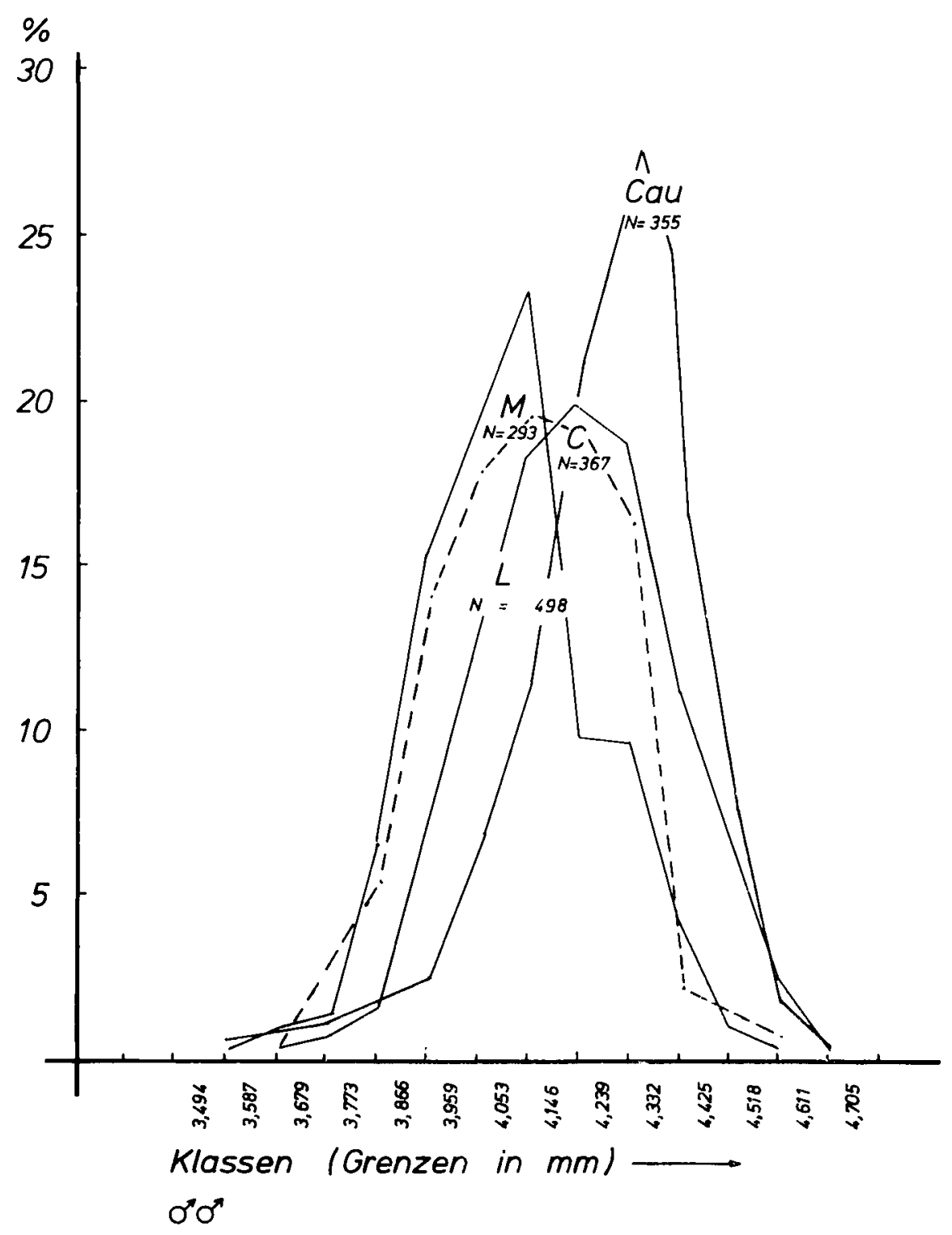

Авв. 3. - Rüssellängen der Drohnen von A.m. mellifica (M), A.m. carnica (C), A.m. ligustica (L--) und A.m. caucasica (Cau).

FIG. 3. - Longueurs du proboscis des mâles d'A.m. mellifica (M), A.m. carnica (C), A.m. ligustica (L---.) et A.m. caucasica (Cau). 


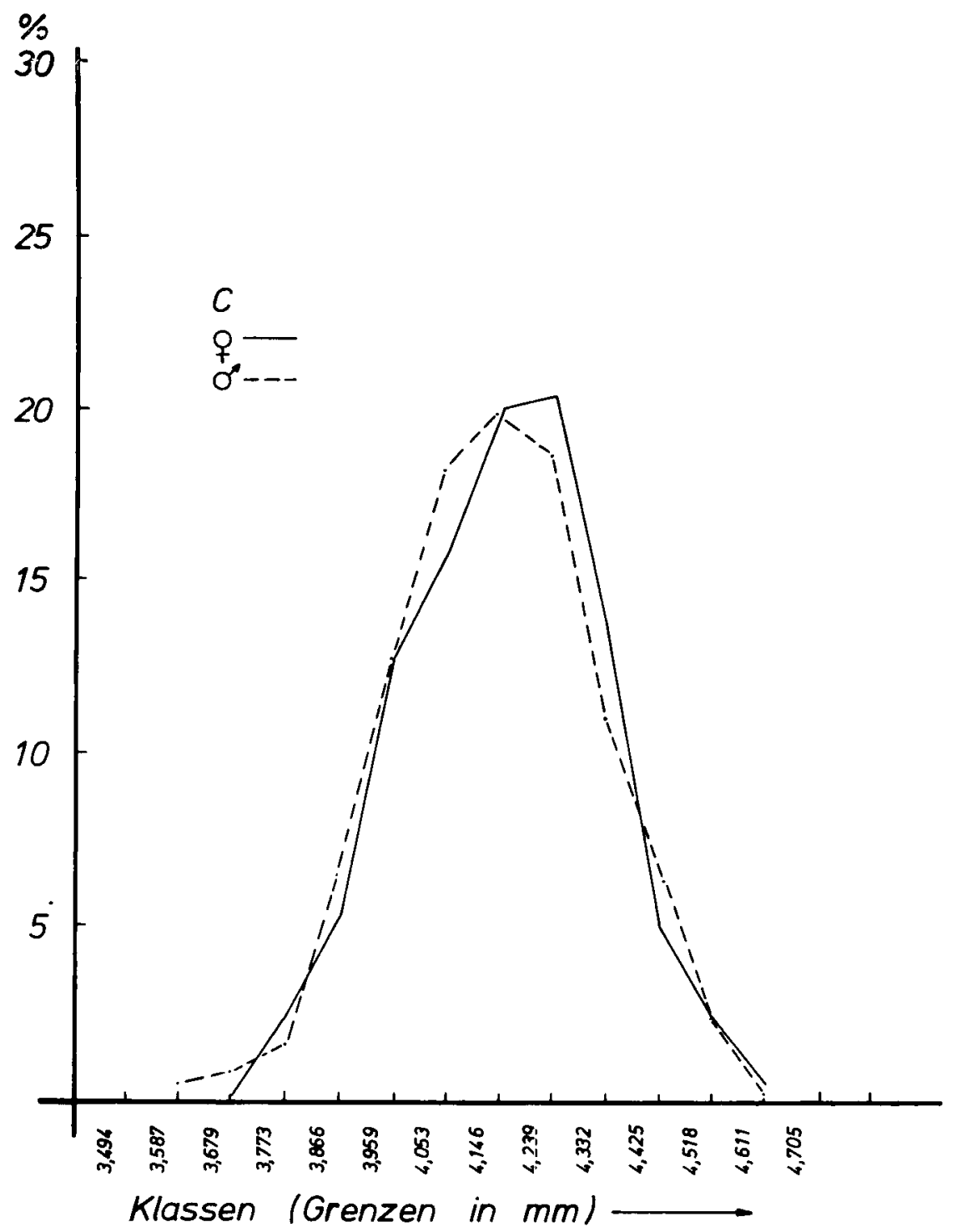

Aвв. 4. - A.m. carnica : Rüssellänge von 1210 Königinnen (-) und 367 Drohnen (- - - )

Fig. 4. -- A.m. carnica : longueurs du proboseis de 1210 reines (-) et de 367 mâles (-- -) 
188

F.-K. BÖTTCHER

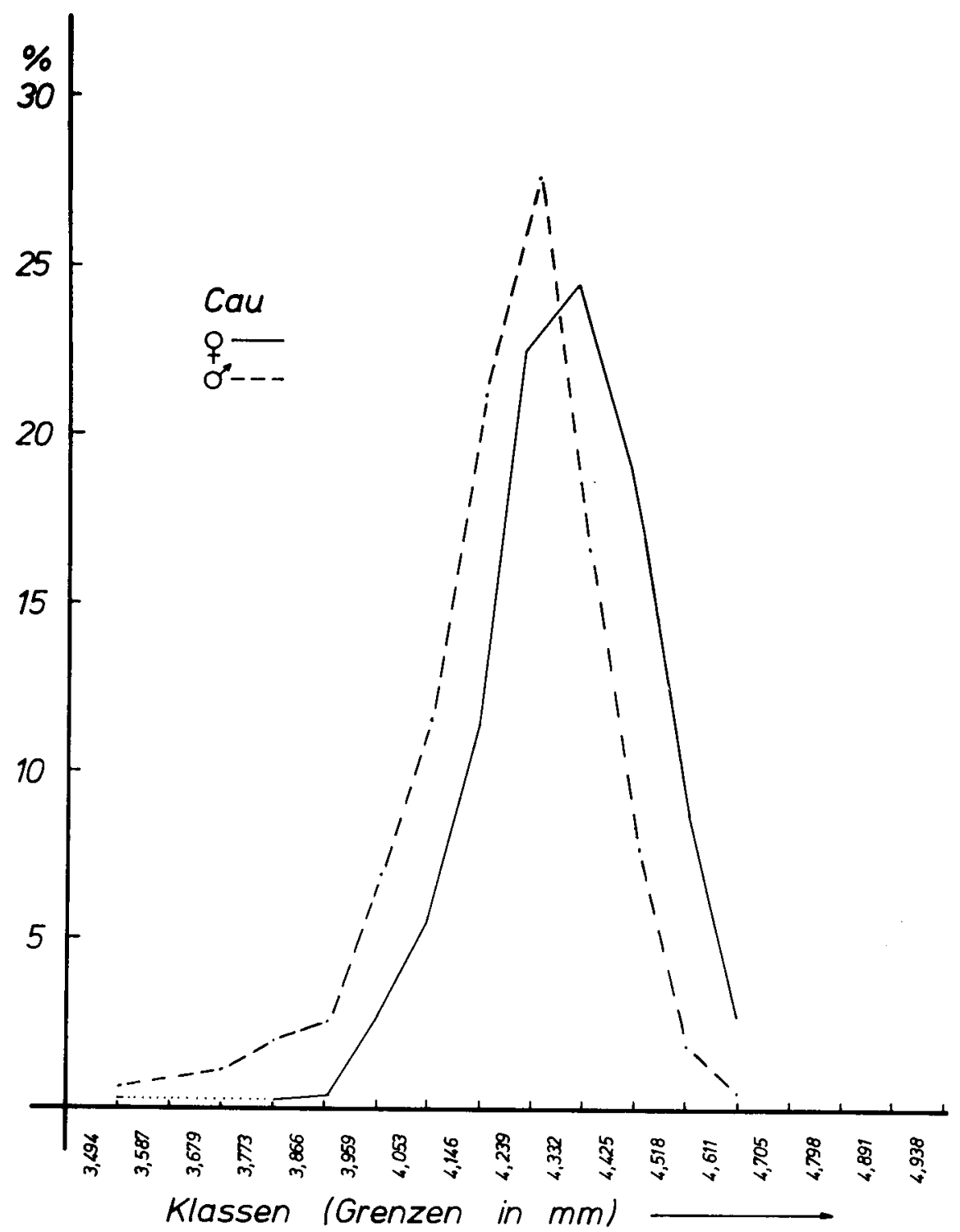

Aвв. 5. - A.m. caucasica : Rüssellänge von 620 Königinnen (一) und 355 Drohnen (- - -

Fig. 5. -- A.m. caucasica : longueurs du proboscis de 620 reines $(-)$ et de 355 mâles (- - 


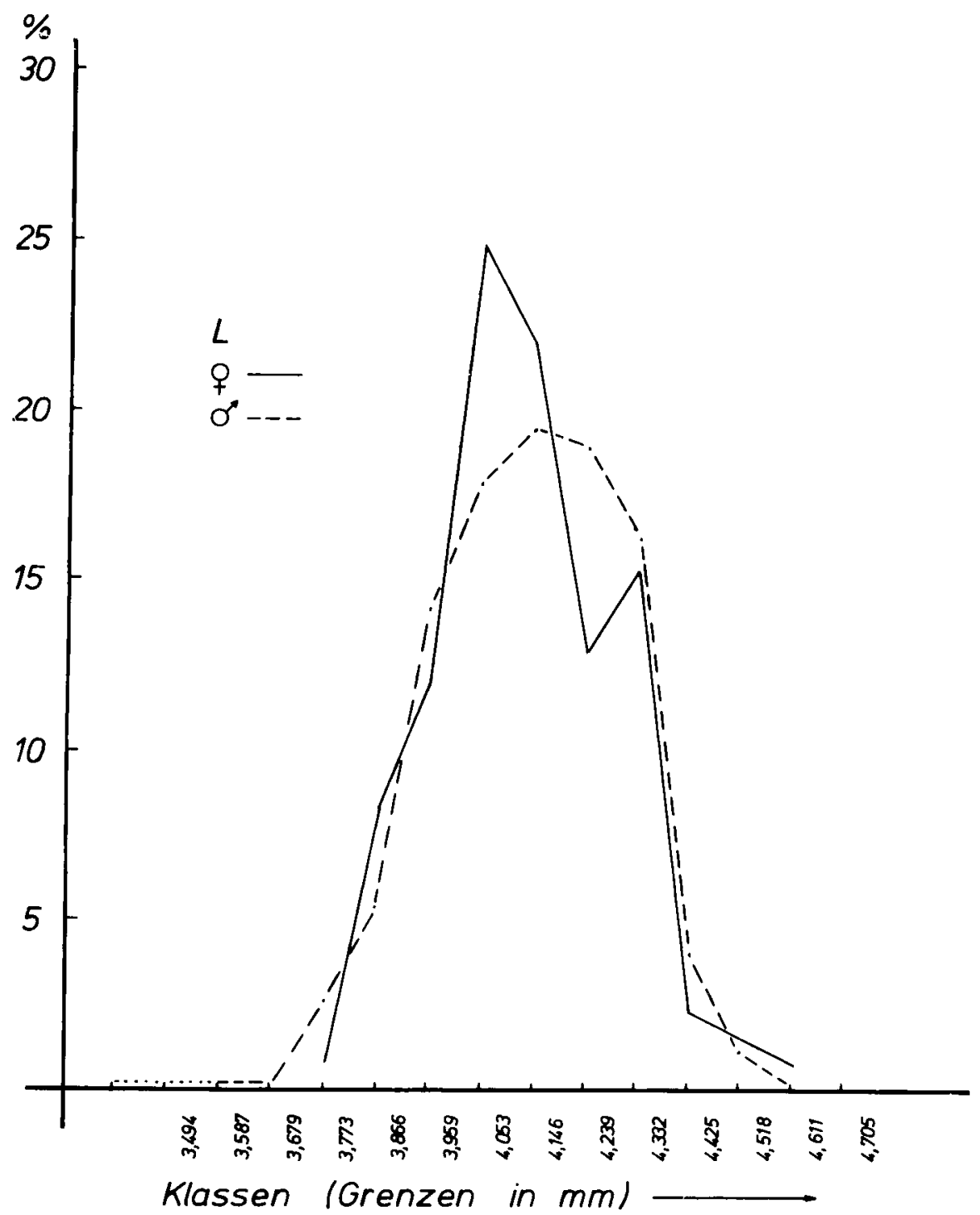

Aв8. 6. - A.m. ligustica : Rüsselänge von 133 Königinnen (-) und 498 Drohnen (-- _ --)

Fig. 6. - A.m. ligustica : longueurs du proboscis de 133 reines (-) et de 498 mâles (- - ) 


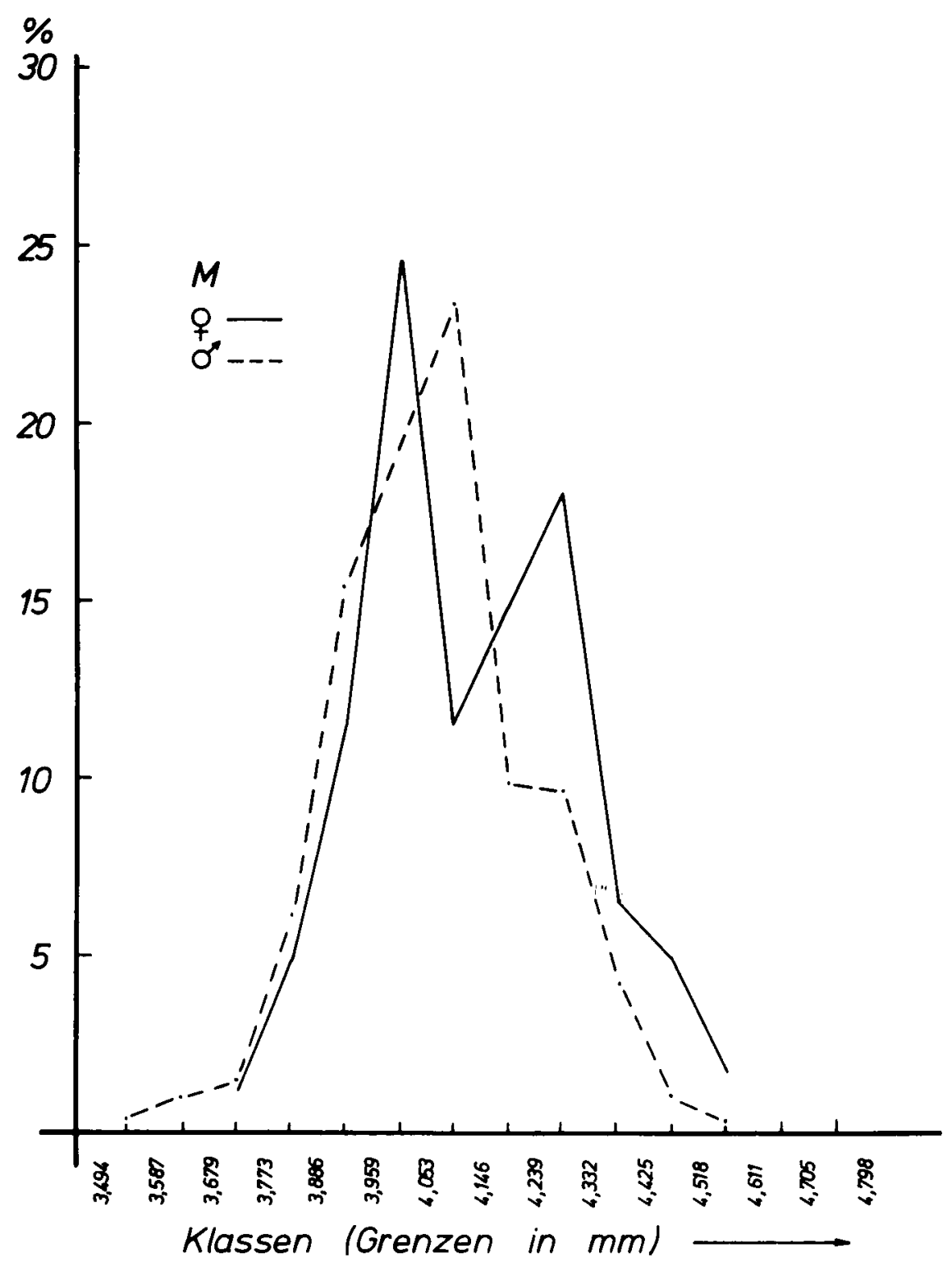

Aвв. 7. - A.m. mellifica : Rüssellänge von 61 Königinnen (-) und 293 Drohnen $(-<-)$ Fig. 7. - A.m. mellifica : longueurs du proboscis de 61 reines $(-)$ et de 293 mâles (- -$)$ 
Von den sich in etwa deckenden Rüsselkurven der Mellifica-Geschlechtstiere (Abb. 7) ist die der Königinnen und andeutungsweise auch die der Drohnen zweigipfelig : Ihr zweiter Gipfel fällt in den Carnica-Bereich (Abb. 2 und 3). Eine Erklärungsmöglichkeit dafür ist, daß die diploiden Königinnen zum Teil aus einer Verkreuzung mit Carnica-Vätern hervorgegangen sind, während die haploiden Drohnen noch nahezu den reinen Charakter ihrer Rasse zeigen. Es kann hierbei aber auch die geringe Anzahl von Meßwerten eine Rolle spielen.

Insgesamt zeigen beim Vergleich der Rüssellänge der 4 verschiedenen Bienenrassen die Verteilungskurven der Meßwerte entsprechend den Mittelwerten einen eindeutigen Zusammenhang zwischen den Rüssellängen der Arbeitsbienen einerseits und der Königinnen und Drohnen andererseits.

\section{B. - RÜSSElmessungen BeI KöNIGINNEN VERSCHIEDENER RASSE AUS UMWELTGLEICHEN ZUCHTEN}

Die rassenverschiedene Rüssellänge der Geschlechtstiere überprüften wir bei Königinnen der Carnica- und der Caucasica-Rasse, die wir gleichzeitig und unter umweltgleichen Bedingungen, d.h. in gleichen Pflegevölkern aufzogen. Da aber ein Pflegevolk nur eine beschränkte Anzahl Königinnen aufzuziehen vermag, bestand die Notwendigkeit, mehrere Pflegevölker zu verwenden, um zu der erforderlich erachteten größeren Anzahl Königinnen zu kommen. Dabei aber befanden sich nur die im jeweiligen Pflegevolk heranwachsenden Königinnen unter gleichen Umweltverhältnissen.

\section{Versuch}

Ich ging von 2 Völkern aus, deren Arbeitsbienen verschieden, aber nicht extrem verschieden lange Rüssel hatten : einem mittelrüsseligen Volk von überwiegendem Carnica-Charakter und einem sehr langrüsseligen Volk der Kaukasier Rasse.

Wie die Abb. 8 zeigt, sind die Rüssel der Arbeitsbienen dieser beiden Völker trotz starker Streuung der Werte des Carnica-Volkes so verschieden lang, daß ihre Variabilitätskurven völlig getrennt voneinander liegen. Die Arbeitsbienen des Carnica-Volkes hatten eine mittlere Rüssellänge von 6,438 mm bei einer Streuung von 6,115-6,731 mm, die des Caucasica-Volkes hingegen von $7,137 \mathrm{~mm}$ bei einer Streuung von 6,873-7,347 mm. Die Differenz zwischen den Mittelwerten betrug somit $0,699 \mathrm{~mm}$.

Im Streben nach einer möglichst großen Anzahl von Rüsselwerten zog ich zweimal Königinnen von diesen beiden Völkern jeweils nebeneinander in 4. Pflegevölkern. Beide Zuchten zusammengefaßt ergab die in Abb. 9 dargestellte Verteilung der Rüssellängen. Ihre Mittelwerte sind, ähnlich wie die 
der Arbeiterrüssel, deutlich voneinander getrennt : 4,141 und 4,339 mm. Aber die Einzelwerte überlagern sich stark. Die Streuung ist groß. Die Überprüfung des Unterschiedes zwischen beiden zusammengenommenen Zuchtergebnissen mit Hilfe des t-Testes ergab trotzdem eine sehr hohe Signifikanz von $\mathrm{t}=8,05^{* * *}(\mathrm{f}=145, \alpha<<0,1)$.

Betrachtet man die beiden Zuchten jeweils für sich (Abb. 10 und 11), so zeigt sich bei beiden ein ähnliches und mit Abb. 9 in den Umrissen übereinstimmendes Bild : Die Werte der Caucasica-Rüssel liegen im Vergleich mit denen der Carnica-Rüssel deutlich im höheren Bereich, dementsprechend auch die Mittelwerte. Die Werte der Carnica-Rüssel lassen jeweils 2 Gipfel erkennen, die auch bei den Säulen der Arbeiterrüssel angedeutet sind (Abb. 8).

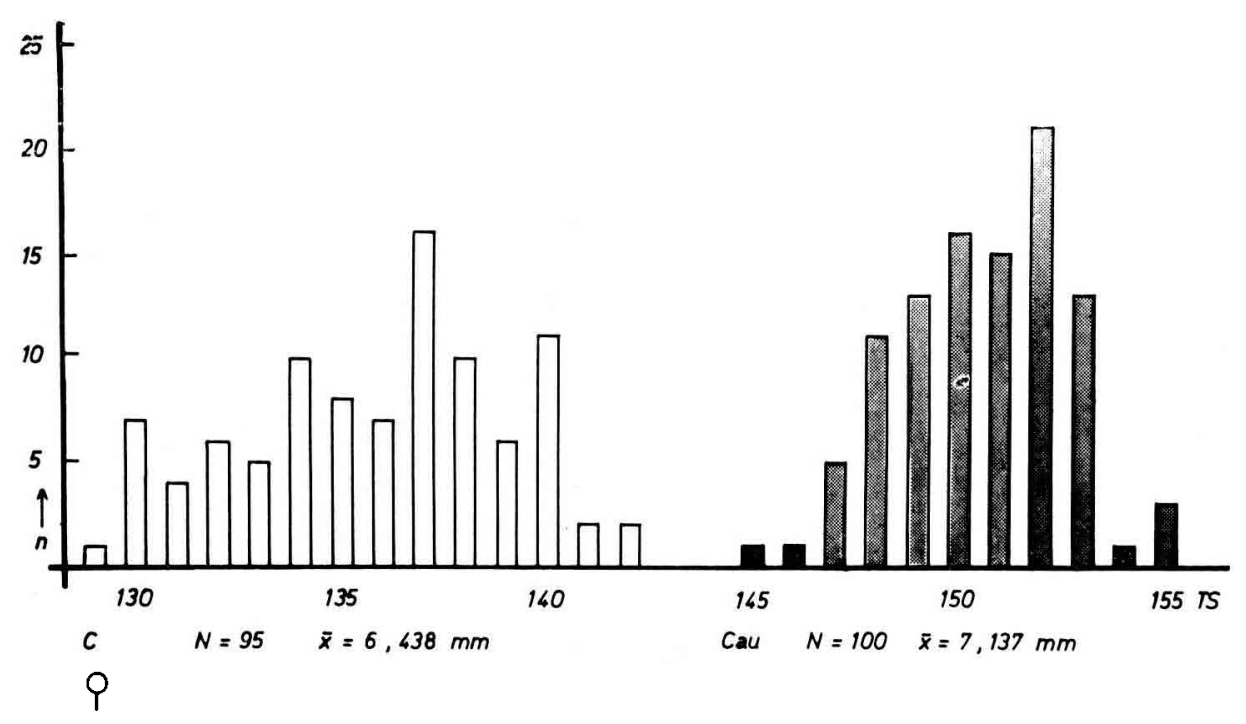

Авв. 8. - Rüssellängen der Arbeitsbienen eines mittelrüsseligen Carnicaund eines langrüsseligen Caucasica-Volkes.

Abszisse $=$ Rüssellänge in Teilstrichen (TS) 1 Teilstrich $=0,04658 \mathrm{~mm}$

Ordinate $=$ Anzahl der Rüssel (n)

$\mathrm{C}=$ Carnica

Cau =: Caucasica

$\overline{\mathbf{x}} \quad=$ Mittelwert

Fig. 8. - Longueurs du proboscis des ouvrières d'une colonie de carnica à langue moyenne et d'une colonie de caucasica à langue longue

En abscisses : longueur du proboscis en tirets (TS). 1 tiret $=0,04658 \mathrm{~mm}$

En ordonnées : nombre de proboscis (n) $\mathrm{C}=$ Carnica

$\mathrm{Cau}=$ Caucasica

$\overline{\mathbf{x}}=$ moyenne

Bei der ersten Zucht in 4 Pflegevölkern (Abb. 10) beträgt die mittlere Rüssellänge der Carnica-Königinnen $4,154 \mathrm{~mm}$, die der Caucasica-Königinnen $4,311 \mathrm{~mm}$, bei der zweiten Zucht entsprechend 4,133 und 4,448 mm. Auch die 
Variationsbreite ist in beiden Zuchten ähnlich. Die Signifikanz für den Unterschied der Carnica- und Caucasica-Rüssel ist bei beiden einzeln betrachteten Zuchten ebenso wie bei der zusammengefaßten sehr hoch $(t=5,96, f=83$, $\alpha<<0,1 \%$ und $\mathrm{t}=6,91, \mathrm{f}=60, \alpha<<0,1 \%$ ).

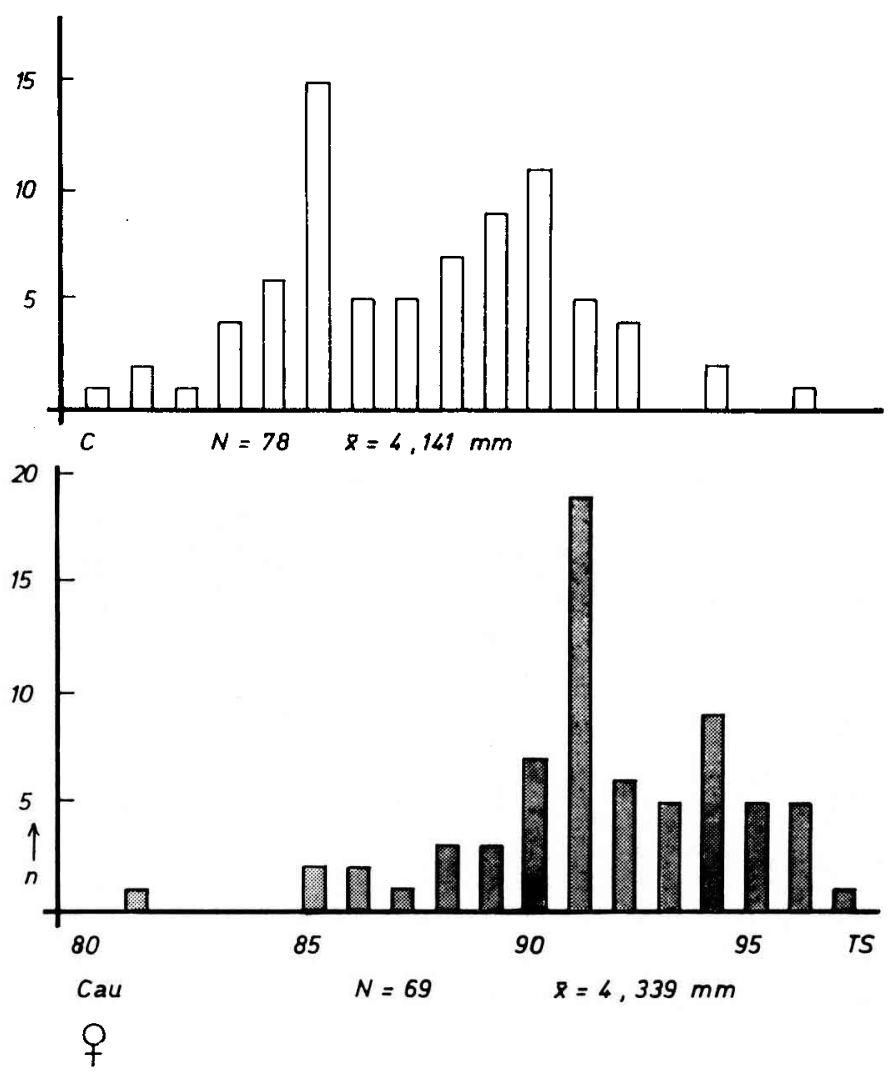

AвB. 9. - Rüssellängen der Königinnen, gezogen von den beiden Völkern der Abb. 8 (1.und 2.Zucht vereinigt)

Abszisse $=$ Rüssellänge in Teilstrichen (TS) 1 Teilstrich $=0,04658 \mathrm{~mm}$ Ordinate $=$ Anzahl der Rüssel (n)

$$
\begin{aligned}
& \mathrm{C}=\text { Carnica } \\
& \text { Cau }=\text { Caucasica } \\
& \overline{\mathrm{x}}=\text { Mittelwert }
\end{aligned}
$$

FIG. 9. - Longueurs du proboscis des reines élevées par les deux colonies de la Fig. 8 (élevages 1 et 2 réunis)

En abscisses : longueur du proboscis en tirets (TS). 1 tiret $=0,04658 \mathrm{~mm}$ En ordonnées : nombre de proboseis (n) $\mathrm{C}=$ carnica

$$
\begin{aligned}
& \text { Cau }=\text { caucasica } \\
& \overline{\mathrm{x}}=\text { moyenne }
\end{aligned}
$$

Bei der zweiten der beiden Zuchten waren die Königinnen entsprechend ihren Pflegevölkern (I-IV) getrennt gehalten worden. Ihre Rüsselwerte konnten 
entsprechend zusammengestellt werden (Tab. 6 und Abb. 12). Die Differenzen zwischen den Carnica- und Caucasica-Rüssellängen sind bei einem Mittelwert von $0,313 \mathrm{~mm}$ klar und deutlich.

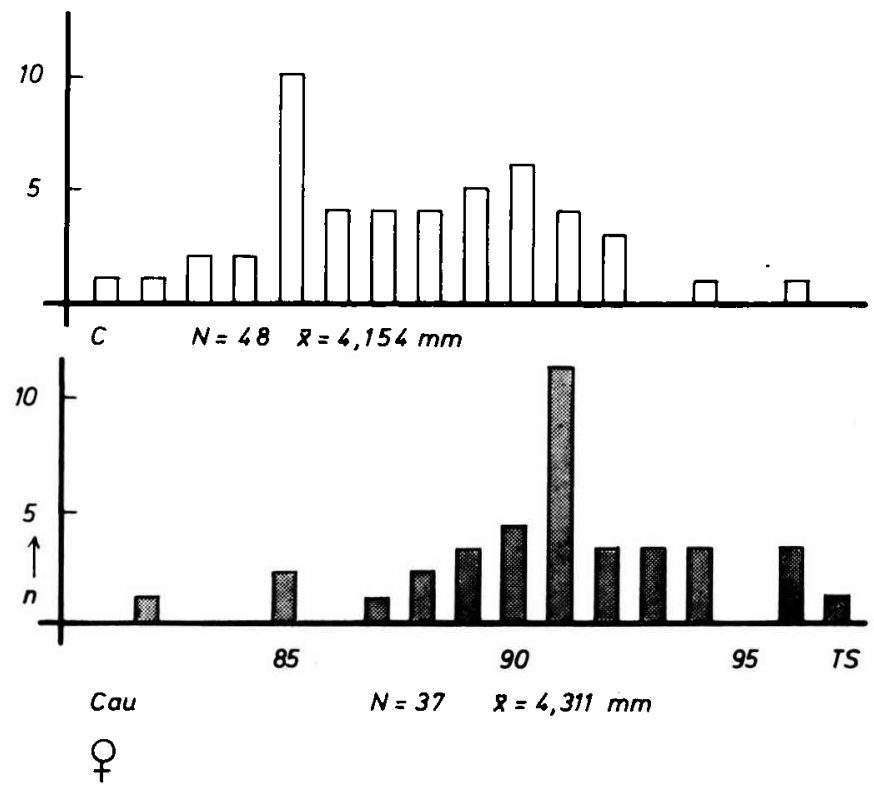

Aв8. 10. - Rässellängen der Königinnen der 1. Zucht

Abszisse $=$ Rüssellänge in Teilstrichen (TS) 1 Teilstrich $-=0,04658 \mathrm{~mm}$

Ordinate $=$ Anzahl der Rüssel (n)

$\mathrm{C}=$ Carnica

Cau - Caucasica

$\overline{\mathrm{x}} \quad$... Mittelwert

FIg. 10. - Longueurs du proboscis des reines du ler élevage

En abscisses : longueur du proboseis en tirets (TS). 1 tiret $=0,04658 \mathrm{~mm}$

En ordonnées : nombre de proboscis (n) C = carnica

$\mathrm{Cau}=$ caucasica

$\overline{\mathbf{x}} \quad$ moyenne

In Abb. 12 erkennt man die starke Streuung der Werte, vor allem die der Carnica-Königinnen. In jedem einzelnen Fall, insbesondere bei den in den Pflegevölkern II und III erzogenen Königinnen, ist die Überlagerung der Rüsselwerte geringer, d.h. erscheinen die Werte besser getrennt, als bei der Zusammenfassung aller. Das mag für verschieden starken Einfluß der Pflegevölker, d.h. für einen Einfluß der Aufzuchtbedingungen auf die Rüssellänge sprechen. Der t-Test ergab statistisch gut bis sehr gut gesicherte Unterschiede zwischen den Rüssellängen der Carnica- und Caucasica-Rasse bei allen 4 Zuchtergebnissen $\left(t_{1}=3,76^{* *}, f=13, \quad 1 \%>\alpha>0,2 \% ; t_{11}=4,06 \%{ }^{* *}, f=11\right.$, $1 \%>\alpha>0,2 \% ; \mathrm{t}_{\mathrm{III}}=5,21^{* * *}, \mathrm{f}=12, \alpha<0,1 \% ; \mathrm{t}_{\mathrm{wv}}=2,98, \mathrm{f}=17, \quad 1 \%$ $>\alpha>0,2)$. 


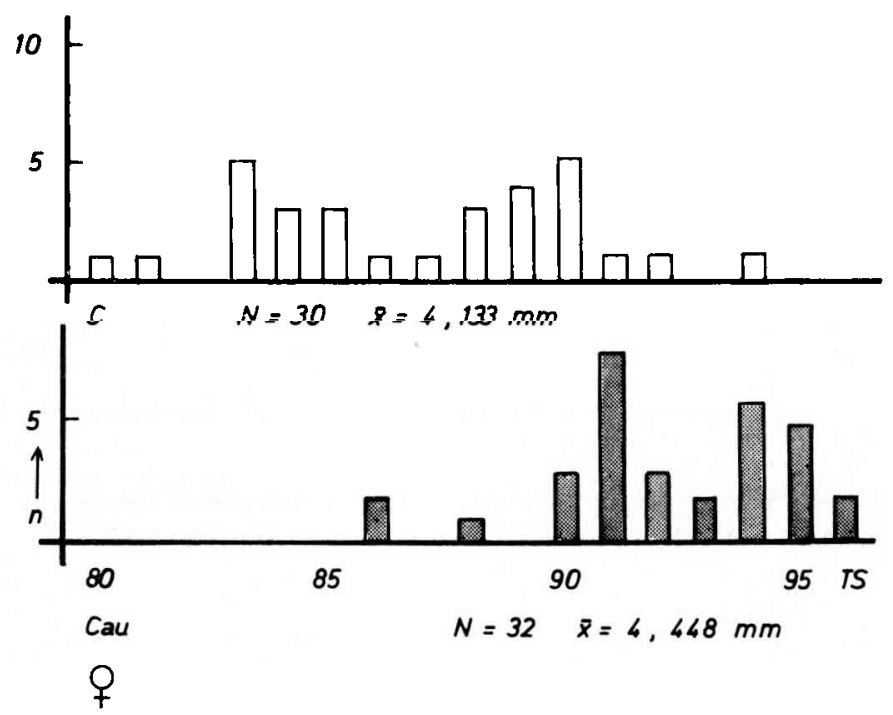

Aвв. 11. - Rüssellängen der Königinnen der 2. Zucht

Abszisse $=$ Rüssellänge in Teilstrichen (TS) 1 Teilstrich $=0,04658 \mathrm{~mm}$ Ordinate $=$ Anzahl der Rüssel (n)

$\mathrm{C}=$ Carnica

$\mathrm{Cau}=$ Caucasica

$\overrightarrow{\mathbf{x}}=$ Mittelwert

Fig. 11. - Longueurs du proboscis des reines du $2^{\mathrm{e}}$ élevage

En abscisses : longueur du proboscis en tirets (TS). 1 tiret $=0,04658 \mathrm{~mm}$ En ordonnées : nombre de proboscis (n) $\mathrm{C}=$ carnica

$\mathrm{Cau}=$ caucasica

$\overline{\mathbf{x}}=$ moyenne

TAB. 6. - Mittlere Rüssellänge von jeweils im gleichen Pflegevolk gezogenen Königinnen verschiedener Rasse (in $\mathrm{mm}$ ).

TABL. 6. - Longueur moyenne du proboscis de reines de différentes races élevées dans la même colonie (en $\mathrm{mm}$ ).

\begin{tabular}{c|c|c|c}
$\begin{array}{c}\text { Pflegevolk } \\
\text { Colonie éleveuse }\end{array}$ & Carnica & Caucasica & $\begin{array}{l}\text { Differenz } \\
\text { Différence }\end{array}$ \\
\hline I & 4,037 & 4,345 & 0,308 \\
II & 4,207 & 4,446 & 0,239 \\
III & 4,112 & 4,489 & 0,377 \\
IV & 4,008 & 4,334 & 0,326 \\
\hline
\end{tabular}



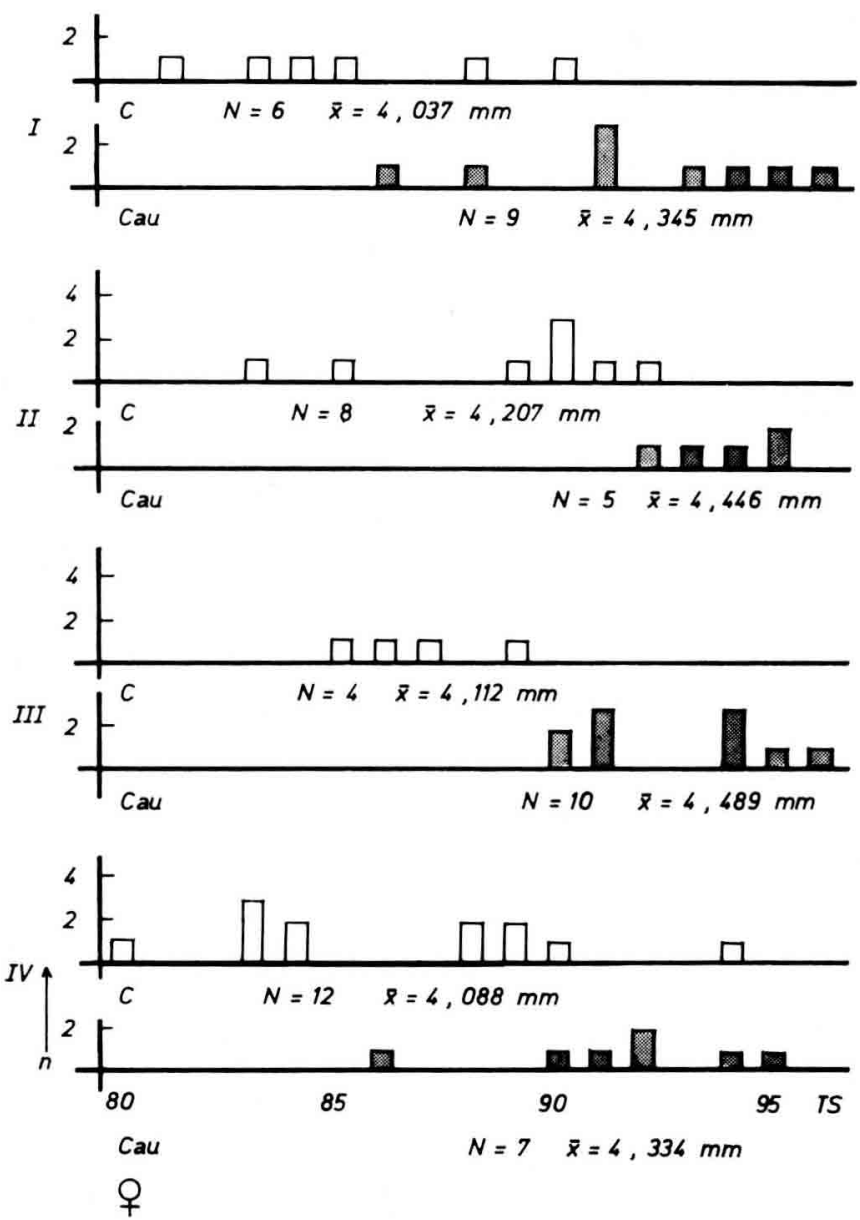

Aвв. 12. - Rüssellängen der Königinnen der 2. Zucht (Abb. 11) aufgegliedert nach den 4 Pflegevölkern Abszisse - Rüssellänge in Teilstrichen (TS) 1 Teilstrich $=0,04658 \mathrm{~mm}$

Ordinate $=$ Anzahl der Rüssel (n)

C - Carnica

Cau - Caucasica

$\overline{\mathbf{x}}=$ Mittelwert

FIG. 12. - Longueurs du proboseis des reines du $2^{\circ}$ élevage (fig. 11) divisé en 4 colonies éleveuses En abscisses : longueur du proboscis en tirets (TS). 1 tiret $=0,04658 \mathrm{~mm}$

Fn ordonnées : nombre de proboscis (n) $\mathrm{C}=$ carnica

Cau - caucasica

$\overline{\mathbf{x}}=$ moyenne 
Somit zeigte sich bei so gut wie jeder der durchgeführten Zuchten, selbst bei nur wenigen Werten, ein eindeutiger Unterschied in den Rüssellängen beider Rassen.

\section{Versuch}

Der Versuch wurde im folgenden Jahr mit 3 Pflegevölkern wiederholt. Die Voraussetzungen waren insofern verschärft, als die Rüsselwerte der Arbeitsbienen der beiden Zuchtvölker der Carnica- und der Caucasica-Rasse dichter zusammenlagen. Die Differenz zwischen den beiden Mittelwerten (6,679 und $6,976 \mathrm{~mm}$ ) betrug nur $0,297 \mathrm{~mm}$, und die Meßwerte überlagerten sich zum Teil (Abb. 13). Die geringe Zahl der Rüsselwerte beim Carnica-Volk $(\mathbf{n}=10)$ dürfte das Bild grundsätzlich kaum beeinträchtigen.

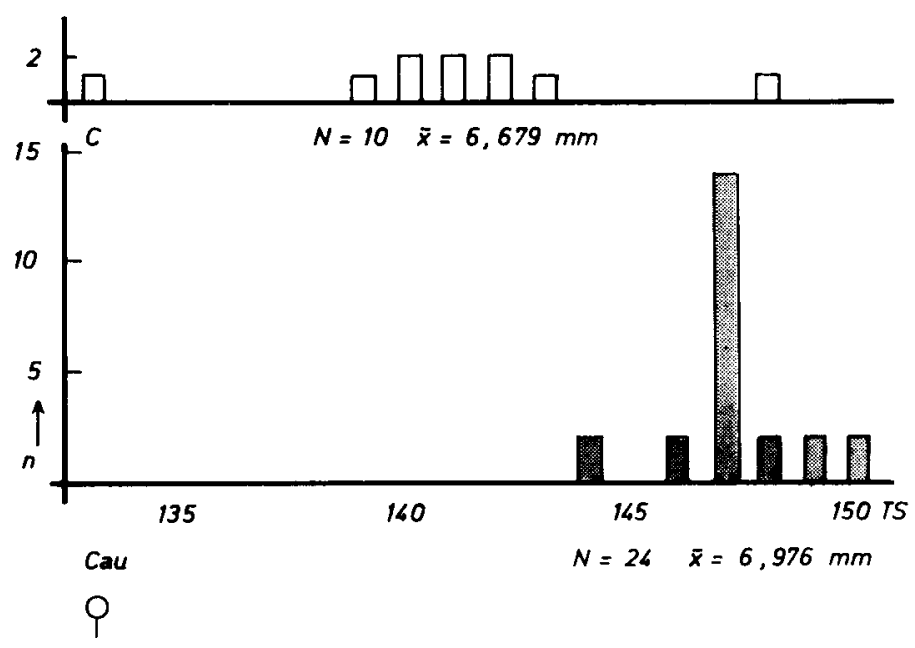

Авв. 13. - Rüssellängen der Arbeitsbienen eines relativ langrüsseligen Carnicaund eines relativ kurzrïsseligen Caucasica-Volkes

Abszisse $=$ Rüssellänge in Teilstrichen (TS) l Teilstrich $=0,04658 \mathrm{~mm}$ Ordinate = Anzahl der Rüssel (n)

$$
\begin{aligned}
& \mathrm{C}=\text { Carnica } \\
& \mathrm{Cau}=\text { Caucasica } \\
& \overline{\mathrm{x}}=\text { Mittelwert }
\end{aligned}
$$

FIG. 13. - Longueurs du proboscis des ouvrières d'une colonie carnica à langue relativement longue et d'une colonie caucasica à langue relativement courte.

En abscisses : longueur du proboscis en tirets (TS). 1 tiret $=0,04658 \mathrm{~mm}$

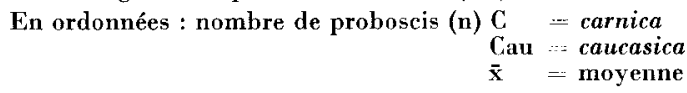

Die Differenz der durchschnittlichen Rüssellängen der aufgezogenen Königinnen war entsprechend geringer (Tab. 7, vgl. Tab. 6). Sie betrug 0,208 mm bei einer mittleren Länge der Carnica-Rüssel von 4,003 und der CaucasicaRüssel von $4,211 \mathrm{~mm}$. Die Längenwerte aller Königinnenrüssel überlagerten sich etwas (Abb. 14), wie bei den beiden Zuchten des ersten Versuchs (Abb. 9- 
11). Ähnlich wie beim ersten Versuch war der Unterschied zwischen den Rüssellängen der beiden Zuchten hochsignifikant $(\mathrm{t}=6,13, \mathrm{f}=39, \alpha<<$ $0,1 \%$.

TAB. 7. - Mittlere Rüssellänge von jeweils im gleichen Pflegevolk gezogenen Königinnen verschiedener Rasse.

TABL. 7. - Longueur moyenne du proboscis de reines de différentes races élevées dans la même colonie.

\begin{tabular}{c|c|c|c}
\hline $\begin{array}{c}\text { Pflegevolk } \\
\text { Colonie éleveuse }\end{array}$ & Carnica & Caucasica & $\begin{array}{l}\text { Differenz } \\
\text { Différence }\end{array}$ \\
\hline I & 3,942 & 4,220 & 0,278 \\
II & 4,013 & 4,266 & 0,253 \\
III & $\frac{4,148}{4,053}$ & $\frac{0,095}{0,003}$ & 0,208
\end{tabular}
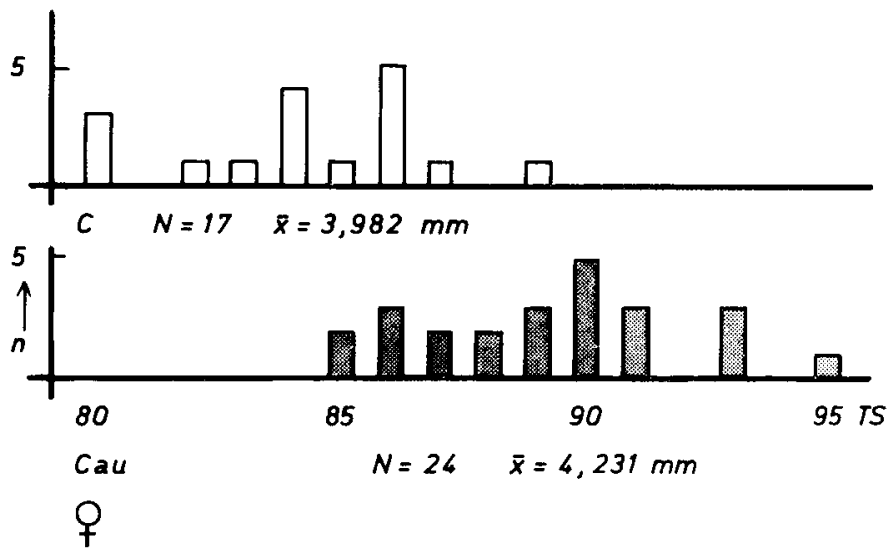

Aвв. 14. - Rüssellängen der Königinnen, gezogen von den beiden Völkern der Abb. 13

Abszisse $=$ Rüssellänge in Teilstrichen (TS) 1 Teilstrich $=0,04658 \mathrm{~mm}$ Ordinate $=$ Anzahl der Rüssel (n)

$$
\begin{aligned}
& \mathbf{C}=\text { Carnica } \\
& \text { Cau }=\text { Caucasica } \\
& \overline{\mathbf{x}}=\text { Mittelwert }
\end{aligned}
$$

Fig. 14. - Longueurs du proboscis des reines élevées par les deux colonies de la fig. 13 En abscisses : longueur du proboscis en tirets (TS). 1 tiret $=0,04658 \mathrm{~mm}$

$$
\begin{aligned}
\text { En ordonnées : nombre de proboscis (n) } & =\text { carnica } \\
\text { Cau } & =\text { caucasica } \\
\overline{\mathbf{x}} & =\text { moyenne }
\end{aligned}
$$

Auch hier ergab sich bei jeder der in den 3 Pflegevölkern gezogenen Königinnengruppen (Abb. 15 I-III) eine den beiden Rassen entsprechende statistisch gesicherte Verschiedenheit der Rüssellänge, trotz geringer Anzahl der Meßwerte. Mit Ausnahme der Rüssel der Königinnen aus dem Pflegevolk III 
mit ihrer extrem geringen Anzahl waren die Rüsselunterschiede bei den Königinnen der Pflegevölker I und II gut und sehr gut gesichert $\left(t_{1}=2,90^{*}, f=12\right.$, $\left.2 \%>\alpha>1 \% ; t_{11}=5,60^{* * *}, f=21, \alpha<<0,1 \%\right)$.

Somit bestätigt sich in beiden Versuchen, daß ebenso wie bei den Arbeitsbienen auch bei den Königinnen der Carnica- und der Caucasica-Rasse ein Unterschied in der Rüssellänge besteht.
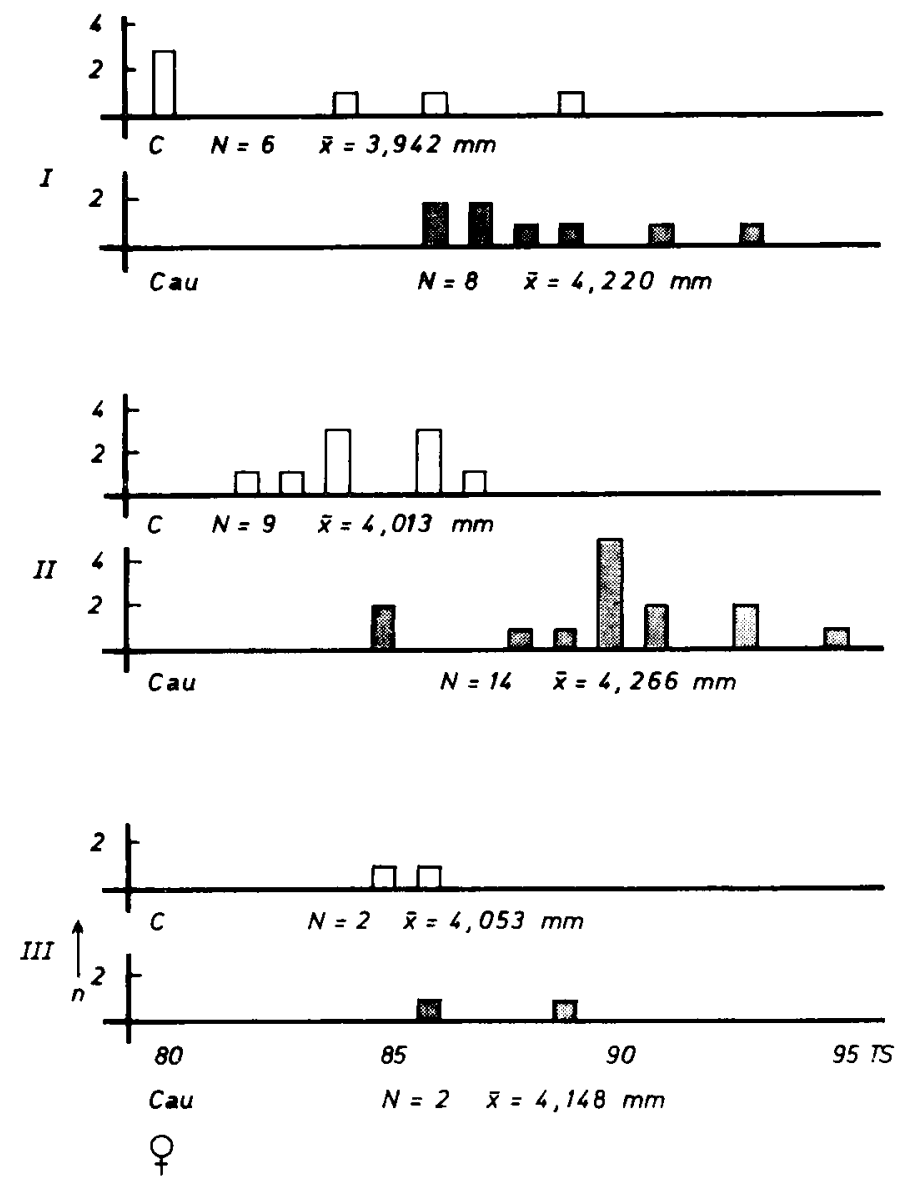

Авв. 15. - Rüssellängen der Königinnen der Abb. 14, aufgegliedert nach den 3 Pflegevölkern.

Abszisse $=$ Rüssellänge in Teilstrichen (TS) 1 Teilstrich $=0,04658 \mathrm{~mm}$

Ordinate $=$ Anzahl der Rüssel (n)

$\mathrm{C}=$ Carnica

Cau $=$ Caucasica

$\overline{\mathbf{x}}=$ Mittelwert

FIg. 15. - Longueurs du proboscis des reines de la fig. 14, divisêes en 3 colonies éleveuses

En abscisses : longueur du proboscis en tirets (TS). 1 tiret $=0,04658 \mathrm{~mm}$

En ordonnées : nombre de proboscis (n) $\mathrm{C}=$ carnira

Cau $=$ caucasica
$\overline{\mathrm{x}}=$ moyenne 


\section{C. - BESTEHT EINE KORRELATION ZWISCHEN DER RÜSSELLÄNGE DER ARBEITSBIENEN UND DER GESCHLECHTSTIERE INNERHALB DER RASSEN?}

Um schnell einen Überblick über die Wahrscheinlichkeit eines solchen Zusammenhanges bekommen zu können, insbesondere zwischen den Rüssellängen der Arbeitsbienen und ihrer königlichen Schwestern, aber auch denen ihrer halbbrüderlichen Drohnen, habe ich in den Tabellen 2-5 die einzelnen Meßergebnisse nach der Länge der Arbeiterrüssel geordnet. Außerdem berechnete ich die Korrelationskoeffizienten (r), ungeachtet der geringen Zahl von vergleichbaren Rüsselwerten, von Königinnen bzw. Drohnen aus demselben Volk wie die Arbeitsbienen. Aus Tabellen und Koeffizienten ergibt sich folgendes Bild :

Bei der Mellifica (Tab. 2) zeigte sich in etwa eine Parallele zwischen der Rüssellänge der Arbeitsbienen und der der Geschlechtstiere, die die Koeffizienten zu bestätigen scheinen $\left(r_{1}=0,96, r_{2}=0,75\right)$.

Bei den Rüsseln der Caucasica-Völker kommt eine negative Korrelation zum Ausdruck $\left(\mathbf{r}_{1}=-0,44 ; \mathbf{r}_{2}=-0,48\right)$, bei den Königinnen hauptsächlich wohl wegen der beiden letzten herausfallenden Werte (Tab. 4, Polen 1 und 2). Aber bei den zwei Kaukasier-Völkern amerikanischer Herkunft scheint sie zu bestehen : kürzerrüsselige Königinnen und Drohnen entsprechen kürzerrïsseligen Arbeitsbienen (s.a. Tab. 1).

Auf keinen bzw. sehr geringen Zusammenhang läßt sich aus den Meß- und Korrelationsdaten der Arbeitsbienen und Geschlechtstiere der Ligustica-Rasse schließen (Tab. $5 ; r_{1}=-0,83 ; r_{2}=+0,37$ ).

Da sich aber in allen Fällen keiner der Korrelationskoeffizienten hinreichend sichern läßt, ist eine mathematisch begründete Aussage nicht möglich. Hauptursache dieser Verschiedenheit ist zweifellos die geringe Zahl der Werte $(\mathbf{n}=3$ bis $\mathbf{n}=11)$. Bei der starken Variabilität der Rüssellänge der Arbeitsbienen (Michailov 1927), der noch breiteren der Königinnen und gar der der wenig untersuchten Drohnen (vgl. GoEtze 1956) muß das besonders ins Gewicht fallen.

Man kann vermuten, daß sich bei größerer Zahl von Meßwerten und bei Aufzucht der zu vergleichenden Individuen unter gleichen Bedingungen vielleicht doch eine gesicherte Korrelation ergibt. Eine absolute Korrelation ist jedoch nicht zu erwarten, da die Rüssellänge der Geschlechtstiere nicht in demselben Maße zunimmt, wie die der Arbeitsbienen (s.S. 177).

\section{D. - KANN MAN NACH DER RÜSSELLÄNGE \\ DER GESCHLECHTSTIERE SELEKTIEREN?}

Die Frage nach der Möglichkeit der Selektion auf Rüssellänge unter Königinnen und Drohnen ist mit den vorliegenden Untersuchungen nicht voll 
geklärt. Goetze (1956) wies schon darauf hin, daß sie bei den Drohnen infolge stärkerer umweltbedingter Variabilität erheblich schwieriger ist als bei den Arbeitsbienen.

Was die rassebedingte Langrüsseligkeit anbelangt, so ist deren Selektion bei Königinnen zweifellos erfolgversprechend. Deshalb wird man bei Rassekreuzungen wahrscheinlich diesen Weg mit Aussicht auf Erfolg gehen können. Aber hinsichtlich der Selektion unter gleichrassigen Königinnen und Drohnen sind noch weitere Untersuchungen mit Bienenmaterial erforderlich, das unter völlig gleichen Bedingungen aufgezogen wurde. Gegebenenfalls müßte unter solchen Voraussetzungen auch die Selektion erfolgen, was bei Drohnen insbesondere nicht immer leicht zu verwirklichen wäre.

Eingegangen im Februar 1977.

Reçu pour publication en fêvrier 1977.

\section{RÉSUMÉ}

La longueur du proboscis des ouvrières d'abeilles est très différente chez les diverses races géographiques d'Europe. A.m. caucasica Gorb. possède le proboscis le plus long, A.m. mellifica L. le plus court. A.m. carnica Polm. et A.m. ligustica Spin. se situent entre les deux.

Les abeilles à long proboscis sont plus à même d'exploiter le trèfle rouge qui possède un calice profond. Certaines années à prédominance de sécheresse, elles apportent en Allemagne une forte miellée inhabituelle. La visite intensive des fleurs influe aussi favorablement sur la production de semences. C'est pourquoi en Allemagne on s'était toujours efforcé d'élever une abeille à langue longue.

Dans cet effort on a sélectionné jusqu'à présent d'après la quantité de miel de trèfle rouge récoltée par les colonies et d'après la longueur du proboscis des ouvrières. Cela faciliterait grandement le travail de l'éleveur, si l'on pouvait également sélectionner d'après la longueur du proboscis des sexués. C'est pourquoi dans le présent travail on a mesuré avant tout la longueur du proboscis des reines et des mâles chez les races citées et essayé de mettre en évidence les relations qui existent entre elles. Puisque les reines comme leurs sœurs ouvrières proviennent d'œufs fécondés et possèdent donc toutes le même patrimoine, la longueur du proboscis des reines doit évoluer parallèlement à celle de leurs ouvrières. Et puisque les mâles proviennent d'oufs non fécondés, on devrait s'attendre à ce que chez eux se reflète clairement le patrimoine de leur mère, qui conditionne la longueur du proboscis.

Les proboscis furent généralement mesurés sur des insectes vivants mais anesthésiés (Bötтснеr, 1961, 1975). De cette façon on travaille très rapidement car on n'a pas besoin de disséquer et préparer. De plus cette méthode offre l'avantage de pouvoir ensuite utiliser pour l'élevage les insectes sélectionnés.

Les résultats sont les suivants :

1. Chez les 4 races étudiées le proboscis des reines et celui des mâles sont à peu près de la même longueur : mellifica 4,144/4,042, ligustica 4,056/4,085, carnica 4,236/4,179, caucasica $4,355 / 4,228 \mathrm{~mm}$.

2. D'une race à l'autre les longueurs des proboscis des reines et des mâles correspondent approximativement à celle des ouvrières, aussi bien pour les valeurs moyennes trouvées (à 
l'exception d'une anomalie chez la race ligustica) que pour la distribution générale. Pour les races citées elles augmentent pourtant un peu moins chez les reines et les mâles que chez les ouvrières. Ceci signifie que la longueur du proboscis est influencée par les facteurs qui déterminent non seulement le sexe et la caste mais aussi la race.

3. Lorsqu'on élève dans des conditions semblables des reines provenant de colonies de carnica et de caucasica avec des ouvrières ayant des proboscis de diverses longueurs, une différence significative apparaît rapidement dans la longueur du proboscis des reines des deux races, même si la longueur du proboscis de leurs sœurs ouvrières ne diffère pas beaucoup.

4. En général on n'a pas pu établir à l'intérieur d'une même race de corrélation entre la longueur du proboscis des ouvrières et celle des sexués. Il serait nécessaire pour éclaircir ce problème d'élever un plus grand nombre de reines dans des conditions semblables.

5. Les résultats acquis prouvent que la mesure de la longueur du proboscis des reines peut aider utilement à sélectionner à partir de croisements de races des souches d'abeilles à langue longue.

\section{LITERATUR}

Alpatov W. W., 1929. - Biometrical studies on variation and races of the honey bee (Apis mellifera L.) The Quaterly Review of Biology 4 (1), March 1929, 1-58.

Bötrcher F. K., 1961. - Eine Methode der Rüsselmessung an der unverletzten Biene. Z.f. Bienenforschung 5, H. 7, S. 249-252, (Apic. Abstr. 412/1962).

Bötтcher F. K., 1962/65. - Rüsselmessung durch Projektion - eine einfache Methode für den praktischen Züchter. Der Imkerfreund 17 (9) 301-302, 1962 (s.a. Der Imkerfreund 20 (3) 83,1965$)$.

Bötтcher F. K., 1975. - Die Rüssellänge von Königinnen, Drohnen und Arbeitsbienen. XXV. Internationaler Bienenzüchterkongreß Grenoble, Programm S. 82-85, Kongreßband S. 296-299.

Сноснцоv B. P., 1916. - Untersuchungen über die Rüssellänge der Arbeitsbiene. Ministerium für Landwirtschaft, Petersburg, 16-4l.

Farcas A., 1939. - Variationsstatistische Untersuchungen des Bienenrüssels. Arch.f. Bienenkde. 20 (6) 216-224.

Giavarini I., 1953. - Ricerche biometriche e morphologiche sui caratteri razziali di Apis mellifica sicula Grassi. Mem. Soc. ent. ital. 32, $129-139$ (Apic. Abstr. 76/1965).

GoETze G., 1930. - Variabilitäts- und Züchtungsstudien an der Honigbiene mit besonderer Berücksichtigung der Langrüsseligkeit. Archiv für Bienenkunde 11 (5/6) 185-279.

Goetze G., 1940. - Die beste Biene. Leipzig.

Goetze G., 1948. - Versuche zur Ausnutzung des Rotklees durch die Honigbiene. Beiträge zur Agrarwissenschaft H. 2, (15 S.).

Goetze G., 1956. - Methodik der Selektion der Honigbiene auf Langrüsseligkeit. Insectes Sociaux $3(2), 335-346$.

Gromisz M., 1961. - Die Variabilität der Rüssellänge der Bienen in Polen (Poln. m. dt. Zus.). Pszczelnicze Zeszyty Naukowe 5 (1) 1-31.

Hejtmanek J., 1960. - Studie über die Beurteilung der Saugrüssellänge bei den Bienen. (tschech. m. dt. Zus.) Vedeke Prace vyzkumneho ustavu vcelarskeho 1 (2) 11-37 (Apic. Abstr. 593/1964).

Van Laere O., und Martens N., 1962. - L'importance de l'abeille domestique dans la production de semences de trèfle commun. Rev. Agric. Brux. 15 (11/12) 1383-1395. (Apic. Abstr. 415/1965).

Lekishyili A. und Khidesheli A. L., 1967. - Characteristics of the main populations of the gray Georgian bees. 21. Intern. Bienenzüchterkongreß Maryland (U.S.A.), Vortrag 18, S. 523 (Apic. Abstr. 694/1967).

Melnichenko A. N., 1962. - Versuch, die Merkmale von Königinnen und Drohnen durch 
Aufzucht in Völkern anderer Rasse zu ändern. Agrobiologiya (1) 55-65 (russ.) (Apic. Abstr. 349/1963).

Michailov A. S., 1926. - Über eine lineare Korrelation zwischen der Rüssellänge der Honigbiene und der geographischen Breite im ebenen europäischen Rußland. Arch.f. Bienenkde. 7 (1) 28-33.

Michailov A. S., 1927. - Der Einfluß einiger Lebenslagefaktoren auf die Variabilität der Honigbiene (Apis mellifica L.) Arch. $f$. Bienenkunde 8 (8) 289-303.

Michailov A. S., 1927. - Über die Saison-Variabilität der Honigbiene. Ebenda, 8 (8) 304-312.

MichaILOV A. S., 1927. - Über den Zusammenhang zwischen dem Umfang der Bienenzelle und dem Umfang des Bienenkörpers und seiner Teile. Ebenda 8 (8) 313-321.

ORösI-PÁL Z., 1934. - Über die Rüssellänge der ungarischen Honigbiene (ungar. m. engl. Zus.). Debrceni Szemle 17 (4) 1-13.

Sковıкош A. S., 1929. - Beiträge zur Kenntnis der kaukasischen Honigbienenrassen I-V. (Russ, m. dt. Zus.), Iswestia Otdela Prikladnoi Entomologii GIOA 4 (1) 1-60.

Vlatkovic B. und KuLINcEvic J., 1958. - Beitrag zu morphologischen Untersuchungen an Bienen in Südserbien. Intern. Bienenzüchterkongreß Bologna-Rom S. 251. 